\title{
Toward virtual design and optimization of a structural test monitored by a multi-view system
}

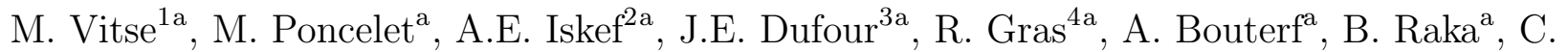

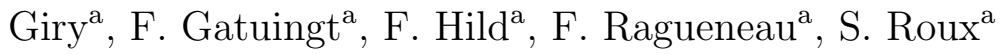 \\ ${ }^{a}$ Université Paris-Saclay, ENS Paris-Saclay, CNRS, LMT - Laboratoire de Mécanique et Technologie, 94235, \\ Cachan, France. \\ Corresponding author: Matthieu Vitse - matthieu.vitse@ens-paris-saclay.fr
}

\begin{abstract}
In order to characterize the mechanical behavior of a reinforced concrete beam-column joint, an experiment is designed whereby different histories of increasing cyclic loads are applied, and the structure deformation (as well as the morphology of apparent surface cracks) are to be studied using multi-view correlation. The complex 3D geometry and the slender nature of the beam and column call for numerous digital cameras to be positioned around the specimen. Assessing the feasibility of image acquisition and estimating a priori uncertainties on multiview correlation becomes a true challenge. It is shown that the recourse to photo-realistic rendering softwares provides the needed tools to perform this experiment design optimization.
\end{abstract}

Keywords: computer vision, mechanical test, multi-view systems, realistic rendering, uncertainty quantification, virtual pre-testing

\section{Introduction}

Scientific visualization is a key aspect in today's science, especially due to the amount of data to process (supplied by more and more powerful clusters of processing units) and the complexity of the phenomena at stake, making it crucial to understand the physics of a simulation or an experiment. Numerous tools are now available to slice, clip or extract subsets of data to provide quasiinstantaneous renderings (e.g., ParaView ${ }^{5}$, Visit ${ }^{6}$, Tecplot ${ }^{7}$ or any kind of custom-made VTK ${ }^{8}$, $I T K^{9}$ or Python ${ }^{10}$ codes). These visualization aspects, so important for post-processing purposes, also appear to be of major interest for pre-processing the experiment, albeit this aspect is seldom highlighted. Because the models to identify become more and more complex, the experimental setup

\footnotetext{
${ }^{1}$ Currently SciViz, France

${ }^{2}$ Currently Razel-Bec, France

${ }^{3}$ Currently University of Texas at Arlington, USA

${ }^{4}$ Currently EikoSim, France

${ }^{5}$ https://www.paraview.org

${ }^{6}$ https://wci.llnl.gov/simulation/computer-codes/visit/

${ }^{7}$ https://www.tecplot.com/

${ }^{8}$ https://vtk.org/

${ }^{9}$ https://itk.org/

${ }^{10}$ https://www.python.org/
} 
becomes more and more diverse and sophisticated (from the geometry of the specimen to be tested [1] to the instrumentation of the test [2]).

This observation applies to the study of reinforced concrete beams and frames, from experiments to modeling, making the prediction of their behavior trustworthy, even for severely damaging conditions. The strength of structures under cyclic or seismic loadings has been the subject of several experimental investigations in recent years $[3,4,5,6,7,8]$. The fair description of joint elements is of major importance when dealing with the load bearing capacity of an entire structure [9]. They are the regions most prone to damage due to the focusing of intense torques transmitted by the connecting beams, and conversely, their softening will control their further degradation and finally the ultimate load the entire assembly can withstand. However, the behavior of the joints between beam and column, and their influence on the overall structure remains the less well-mastered element due to the complexity of the assembly (steel reinforcement bars, anchorage and stirrups) and the occurrence of several coupled physical phenomena (e.g., damage, crack initiation, growth and arrest, debonding of concrete from steel reinforcement, friction, wear of crack surfaces, role of debris [10]).

The lack of exhaustive experimental data hinders rapid and trustworthy progress. One major difficulty in performing experiments on such joints is the expected complexity of their behavior, with localized damage in the form of cracks and their closure [11, 12]. Moreover, the joint is intrinsically a 3D structure that cannot be easily simplified using beam theory. Resorting to simpler mechanical tests according to sub-structuring assumptions $[13,14,15,16]$ may be limited in terms of representativity.

One way to circumvent those difficulties is to perform full-field measurements as they can provide a complete picture of the joint and its connecting beams [17]. Yet, even if the geometry and loading are designed to be planar, there is no chance that through the damaging process, this flatness is preserved. It is very likely that symmetries will be broken and spurious flexural or torsional modes may appear. Rather than disqualifying the experiment as soon as these features appear, it is more attractive (although challenging) to deal with them adequately. Henceforth, the experiment calls for multi-view systems to capture the 3D kinematics of the surface [18]. In addition, it is favorable to observe the joint, the column and the beam over different faces, and also at different scales [19] to capture both the detail of the joint degradation and also the large scale deformation of the beams in order to characterize the loading conditions using beam theory [20]. Because these objects are slender in general, a global view will not have the sufficient spatial resolution to capture minute details. These different requirements and constraints lead to a highly sophisticated setup, as exemplified in Figure 1, namely, a complex T-shaped specimen (1), attached to a machine having a complex geometry (e.g., hexapod $^{1}[21]$ ) and many mobile parts $(2)$, and monitored by several cameras (3).

\footnotetext{
${ }^{1}$ https://en.wikipedia.org/wiki/Stewart_platform
} 


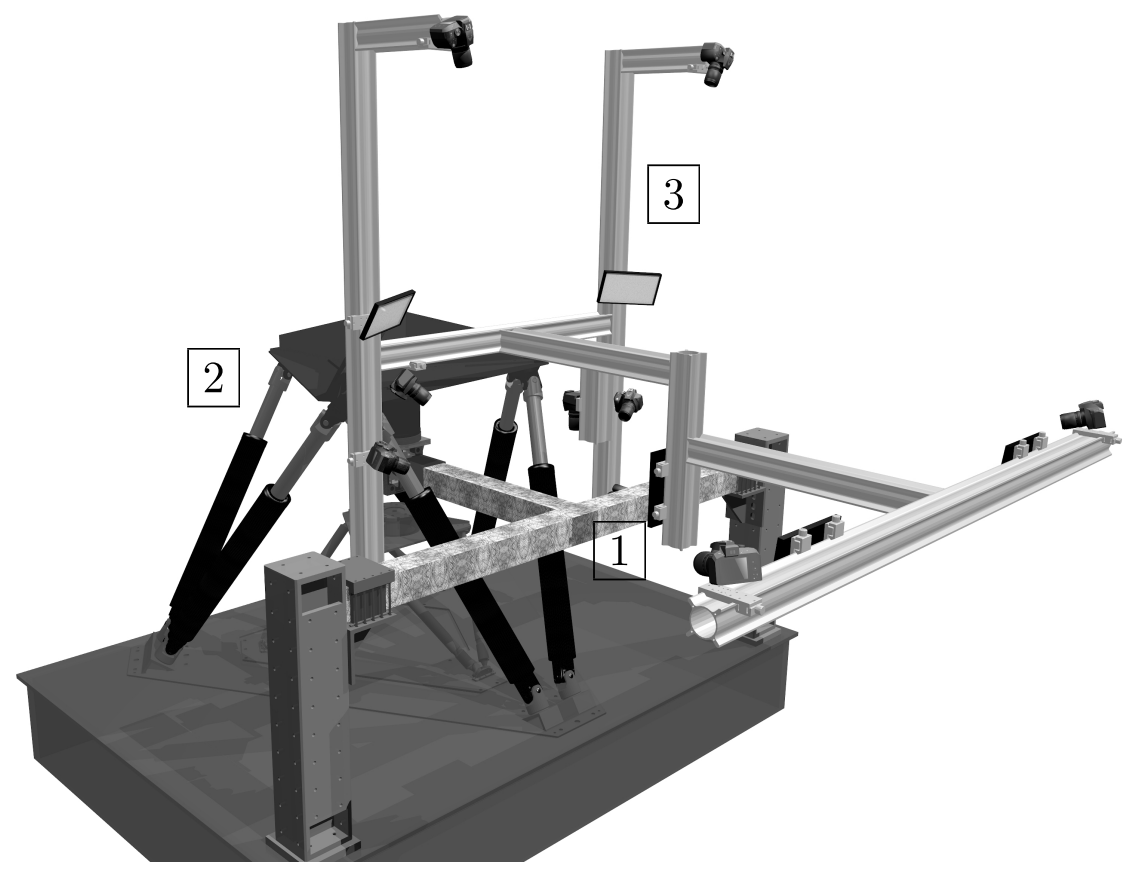

Figure 1: 3D rendering of a complex experimental setup. (1) Beam-column reinforced concrete assembly; (2) Stewart platform (hexapod structure); (3) Acquisition setup (digital cameras, LED panels and supporting system)

The conclusion is that ideally many cameras are to be used simultaneously (with a proper synchronization) in order to fully characterize the kinematics of a joint loaded up to realistic levels (e.g., representative of seismic tests). Consequently, a lot of freedom (as well as many constraints) is involved in the global setup of such an experiment, including the structure design, the loading device and the imaging cameras. Rather than resorting to a "trial and error" procedure to position the cameras, the main objective of this paper is to show that this kind of experiment can be pre-designed using powerful 3D-rendering softwares.

The movie industry has been using such techniques for a long time. First used in theaters in order to stage actors, this step was called "blocking" and performed using mock-ups with simple geometries [22]. Prior to the nouvelle vague ${ }^{1}$ and its dedicated light-weight cameras, the camera location / motion was also a challenge in itself, so that blocking was used to anticipate potential issues relative to the position and motion of both the cameras and actors, especially in the case of crowded or cramped spaces. Last, the communication of visual aims to different people in a very accurate manner is a complication that traditional storyboards could not completely handle ${ }^{2}$. Later computers were used to perform off-stage blocking more accurately ${ }^{3}$. The blocking technique is now

\footnotetext{
${ }^{1}$ French new wave, refers to a group of film makers from the 1950s and the 1960s who were linked by their self-conscious rejection of the traditional movie culture (industry organization, topics or techniques). https://en.wikipedia.org/wiki/French_New_Wave.

${ }^{2}$ This is the reason why film directors want to see exactly "as" the camera, e.g., Stanley Kubrick adding a video camera to the film camera in his 1975 movie Barry Lyndon[23].

${ }^{3}$ The first still wireframe computer-aided design (CAD) images started to be used in the late 1970s to replace traditional blocking, and by the beginning of the 1990s moving wireframes appeared (as during the title sequence of Tim Burton's Batman returns). A decade later, softwares dedicated to this new step called
} 
widely used for 3D computer animation movies, for which key poses (also known as keyframes) are created to establish the timing and placement of the characters, as well as to avoid non-realistic motions.

The requirements of the (animation) movie industry are not those of experimental mechanics. However, visualization softwares are naturally accurate in their geometrical rendering, and this property can be extremely beneficial to the design of complex mechanical tests including the optimization of camera positioning. In the following, a professional free and open-source 3D computer graphics software, Blender [24], is used. Additionally, one looks for very realistic synthetic images of the surface coverage, from which displacement measurements can be mimicked with a specific multi-view correlation software. Blender includes actual surface patterning texture, lighting variations and even representative noise. Therefore, the measurement uncertainties can be evaluated in quantitative terms, and accordingly employed in order to optimize the test. Using a real case study, where the experiment has been performed after having been pre-designed, the focus of the paper is to highlight new possibilities offered by quantitative 3D-rendering softwares, and the added value they can bring.

The experimental challenges exemplified in reinforced concrete structures are in fact representative of a much broader class of applications where industrial parts or even full structures, assemblies of various components, with their real geometry and connections, are subjected to representative (i.e., severe) accidental conditions and loadings. Doubtlessly, a quantitative pre-design of a full experimental setup, where 3D virtual rendering tools can be exploited for predicting realistic multi-view correlation performances, will become an essential step in the future practice of complex experimental tests.

After presenting the considered experimental setup in Section 2, image acquisition is discussed together with the modeling of the experimental geometry within Blender in Section 3. This software produces synthetic images that can be processed in order to validate a priori the feasibility of the experiment in terms of surface coverage or possible shadow obstructions. These features, which are introduced in Section 4, lead to a validation of the experimental design and its optimization prior to its construction. Sections 5 and 6 open the discussion more broadly to the pre-design of complex mechanical tests as an emerging trend to be promoted through innovative tools. This evolution is called for but generic processes for the optimization of such complicated multi-camera setups are to be elaborated, and 3D virtual rendering softwares are very convenient for assessing quantitatively the expected performance of the displacement measurement system, through the evaluation of (displacement) uncertainties (Section 5), which opens a general discussion on the possible input/output of Blender in experimental mechanics (Section 6).

\section{Experimental setup}

\subsection{General framework}

Joint elements exhibit complex $3 \mathrm{D}$ behavior when subject to transverse loadings on the structure (e.g., wind, earthquake [10]). In order to address the issue of thorough characterizations of their response to severe loading intensity and representative mode-coupled loadings, with arbitrary history

\footnotetext{
"pre-visualization" (e.g., FrameForge 3D Studio ${ }^{4}$ ) enabled every of the previously listed points to be checked. It is worth noting that these tools also allow one to make the link between, and merge with, live action and computer-generated imagery (CGI) animation in very intricate manners (as for the long take scene of David Fincher's Panic Room). Numerical accuracy aside, merging CGI and live action can be somehow paralleled with experimental / computation comparisons.
} 
of forces and torques transmitted by the connecting beams, the loading path prescribed to the structure should be at the same time complex and precisely controlled.

In the field of earthquake engineering, the 3D behavior of structures are mainly investigated using a reaction wall or a shaking table [25]. For reaction walls, the loadings are limited to one or two directions according to the number of actuators used to reproduce the earthquake loadings [15, 16] . For shaking tables, where loadings are inertia mediated, getting a full 3D control of the loading applied to the structural element is challenging, as it naturally involves dynamic measurements at a fast rate $[8,26]$.

To circumvent these issues, it is legitimate to consider quasi-static loading conditions so that time per se does not play a significant role. This simplification bears no significant limitations to the sought behavior (except for time-dependent mechanisms). In line with the previous comments, it is important to be able to apply a complex combined loading involving several degrees of freedom. The choice of using a hexapod machine (Figure 2) offering six degrees of freedom appears to be particularly attractive $[27,28,29,30,31,32]$. However, not all six degrees of freedom will be utilized at first, as using only two of them with a non proportional loading history is already quite challenging. Moreover, the same setup can be further extended to reach a higher complexity.

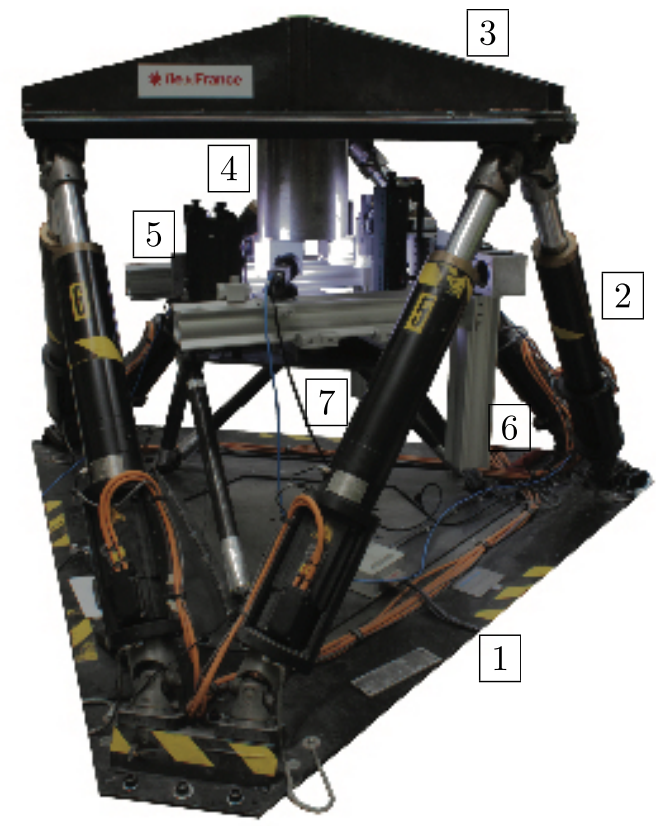

Figure 2: Hexapod testing machine. (1) Base, (2) actuators, (3) moving platform, (4) upper end-effector, (5) area for specimen, (6) optical setup in close configuration with two cameras, (7) load cell with a hexapod structure [21], which was not used in the present case.

The geometry of the chosen structure to be tested is the simplest joint element, namely, a beamcolumn (i.e., T-shaped) structure (14 in Figure 3). Prescribing forces/displacements at the end of the beam with the hexapod (11) while the two ends of the column are clamped (13) is a way to generate a loading on the joint with reasonable balance between complexity and feasibility. The specimen was designed to fit the hexapod capacity according to a numerical model and to exhibit shear failure in the joint region before yielding of beam or column reinforcement bars (subassembly without stirrups in the joint panel).

In terms of modeling, a classical way to take into account the nonlinear response of frame 
structures and joints is to concentrate the nonlinearities at a point, in a so-called "macroelement" $[33,34,35]$ or in a 3D reduced area coupled with simple elements like beams [36]. Generalized strains (i.e., elongations, curvatures and twist) are functionally related to generalized stress (i.e., internal forces and moments) and their complete histories. The nonlinearities are concentrated at the point or the reduced volume where the macro-element stands whereas the remainder of the structural elements (i.e., beams, columns) behave linearly. This challenge is well-defined in the framework of two-scale asymptotic expansions [36].

In order to identify the behavior of the equivalent macro-element, one should get access to the generalized strains and stresses in the elastic portions to be able to extrapolate up to the boundaries of the macro-element. As a consequence, specific strategies are to be developed in this work to provide a measuring system based on elastic beam theory in order to measure the relevant kinematic variables along beam elements, and to perform a trustworthy full 3D extrapolation consistent with the theoretical asymptotic expansion procedure. Because the kinematics is $3 \mathrm{D}$, it is necessary to combine at least two different viewpoints to achieve a 3D (surface) measurement of the displacement field [18]. However, this statement is to be repeated for all beam sections and columns connected to the joint. Moreover, as damage may occur on the entire apparent free surface, it is necessary to have multiple complementary views around the setup, and thus many cameras are to monitor the experiment. The overall setup is technically complex (Figure 3).

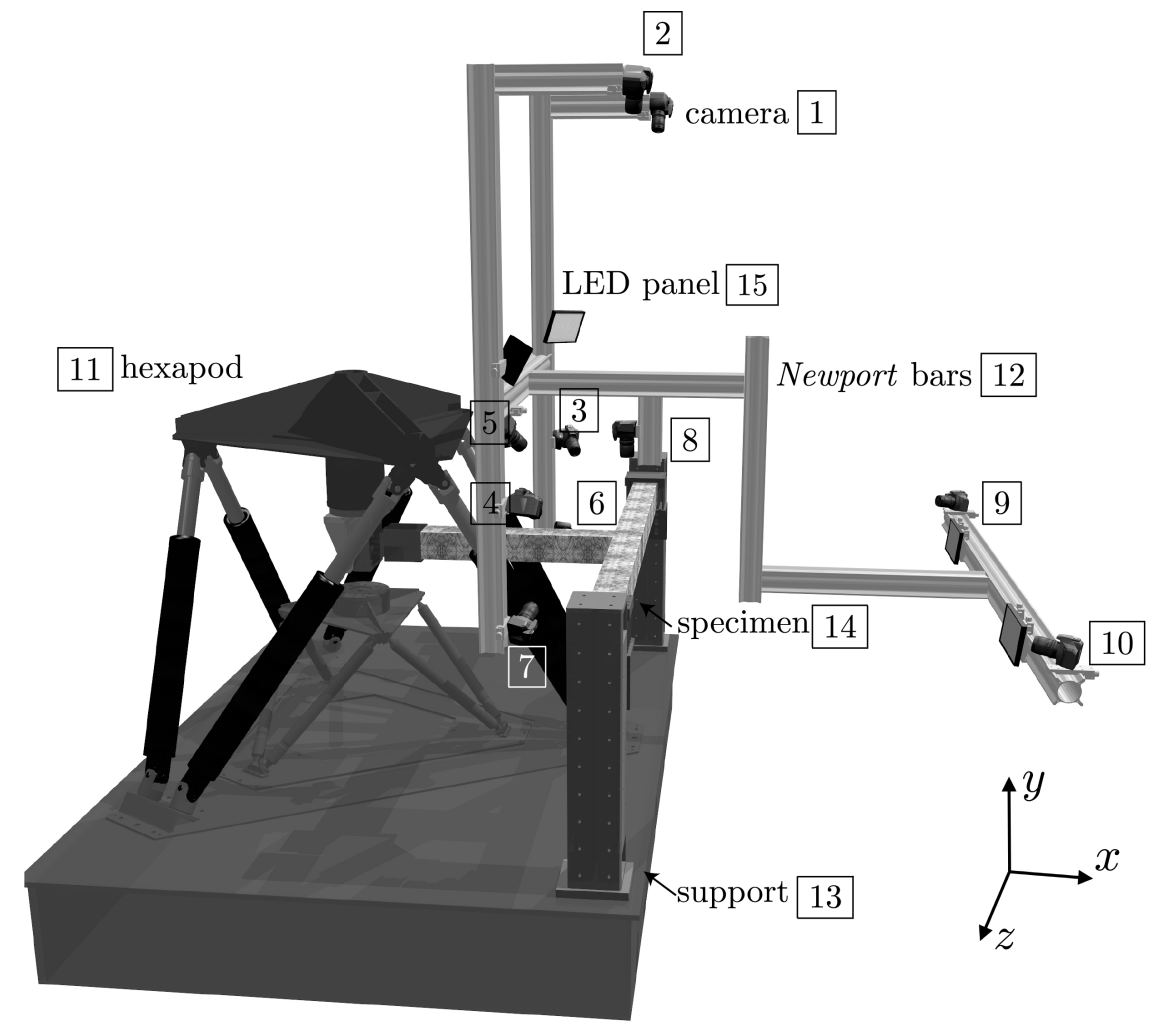

Figure 3: View of the entire setup. (1-10) cameras, (11) hexapod device, (12) Newport bars for supporting the cameras, (13) support of the specimen, (14) reinforced concrete specimen, (15) additional lighting sources.

\subsection{T-shaped specimen}

The length of each half column is equal to $1.14 \mathrm{~m}$, and that of the beam $1 \mathrm{~m}$, the cross section of the column and beam being $120 \times 120 \mathrm{~mm}^{2}$, the joint is a $120 \times 120 \times 120 \mathrm{~mm}^{3}$ cube. It is made of Portland cement with usual reinforcements and several high strength steel bars (anchored in the 
joint regions by $90^{\circ}$-hooks) for longitudinal reinforcements in the column and beam sections. For more details the reader is referred to Ref. [2].

The surface pattern necessary for the digital image correlation (DIC) algorithms [18, 37], which will be described in more details in Section 5, is obtained by first applying a paint coating, in order to get a white background, then a combination of stencil technique leading to a pattern of $2 \mathrm{~mm}$ in diameter black dots and finally black manual brush strokes in order to break the regularity of the pattern (Figure 6(b)).

\subsection{Loading setup 8 loading paths}

The testing machine is a hexapod (Figure 2), i.e., a 6-axis parallel machine. Apart from very few exceptions [27, 28, 29, 30,31,32], this type of machine is still rarely used for material or structural testing, although it offers very versatile and complex loading capabilities. Using six electromechanical actuators, it allows for an overall translation (resp. rotation) range about $\pm 250 \mathrm{~mm}$ (resp. $\pm 22^{\circ}$ ) in every direction (the actual range of translation and rotation is complex because of the non-cartesian parallel structure of the machine [38]).

The machine is equipped with a custom-made six-axis load cell that is fixed onto the mobile platform. It is designed to satisfy the requirement of the intended experiment with a $27 \mathrm{kN}$ (resp. $25 \mathrm{kN}$ ) capacity along the $y$ and $z$ directions, (resp. $x$ ). Similarly, torques can be measured up to $0.6 \mathrm{kN} . \mathrm{m}$ (resp. $11.3 \mathrm{kN} . \mathrm{m}$ ) along $y$ and $z$ (resp. respectively $x$ ). As seen in Figure 3, the specimen is supported in the horizontal position, with each end glued inside steel helmets that are attached to two metal columns (13 in Figure 3) fastened to the steel slab. The last helmet is connected to the load cell through a spherical joint with an initial zero clearance.

The specimen is loaded with a combination of horizontal $\left(\mathbf{u}_{y}\right)$ and vertical $\left(\mathbf{u}_{z}\right)$ translations at the end of the beam using different loading histories (one example is shown in Figure 4). They aim to investigate states that can be encountered by a reinforced concrete joint during earthquake loading (here in quasi-static conditions), and in particular the coupling between different crack patterns developed in different directions [39].

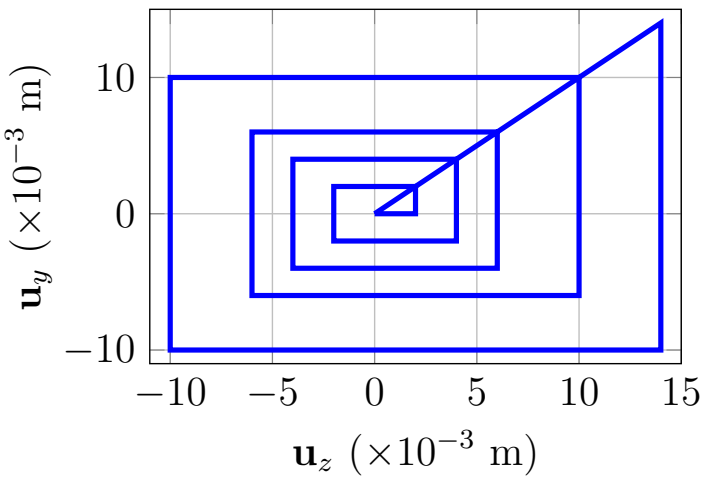

Figure 4: Example of a complex multi-axial loading history applied to the end of the reinforced concrete column. $x$ is the direction of the column, meaning the displacement is prescribed on a plane normal to the structure to trigger different bending modes at the beam-column joint.

\section{Image acquisition and modeling}

As explained in the previous sections, the loading setup itself is complex. Consequently, the requirements of the kinematic measurement setup are as follows:

- because several cameras are to be used, they must be simultaneously triggered; 
- the cameras must capture different faces of the specimen, namely, upper, lower, back and internal ones;

- the camera position and orientation are set to perform multi-view correlation (i.e., at least two cameras must capture the same region), but also with some special requirements such as a minimum angle between the two optical axes;

- the different parts of the setup (including cameras and lighting) must not overshadow some areas of the specimen from the cameras;

- the regions of the specimen monitored by the cameras must be uniformly illuminated.

To address the above specifications, it was decided to use several digital single lens reflex (DSLR) cameras because of their small dimension and high definition. Several powerful light-emitting diode (LED) panels were also available, which were chosen for their rather non-directional lighting properties. A frame made of Newport X95 rails (12 in Figure 3) was chosen for its high modularity and stiffness, equipped with Manfrotto 3-way heads to precisely turn the cameras in the appropriate direction. The main question to be answered is: how to choose and optimize the location, orientation and lens of each camera?

The calibration of numerous cameras may be extremely time consuming and makes the testing machine unavailable for quite a long time. To reduce this cost, a novel approach to design the experiment is tested herein, which is based on the use of a 3D-modeling software. The testing machine and the specimen (including its fixtures) can be described accurately from their CAD descriptions, which are readily available. The environment of the experiment itself can be captured from pictures. The addressed question is to set up virtually the experiment from the available data and find "accurate" viewpoints for the cameras for correlation purposes.

The qualification of "accurate" involves a number of issues detailed below, including the adequacy of surface coverage (on the parts where multi-view correlation is to be used, namely, at least two cameras should monitor the various surfaces of interest), the absence of obstacles masking part of the interesting field of view, the adequate definition of each camera (and hence the choice of lenses), the possibility to position the cameras at the required locations, the lighting setup excluding unfavorable shadows. An even more challenging target is to evaluate a priori the uncertainties expected from multi-view registrations, and hence finally optimize the overall setup under the above mentioned constraints. Because the considered strategy is solely based on modeling, the expression "virtual design" is used.

Central to the approach is the recourse to an open-source 3D-modeling and rendering software (Blender). Although its main field of application is animation movies or aesthetic 3D-renderings [24], it has the capability of reproducing very accurately 3D scenes in a quantitative fashion (i.e., no limiting geometric approximations are included). Hence the photo-realistic rendering is the main motivation for using this software. Additionally, its versatility, its flexible interface, and its material and texture modeling features are very convenient and powerful characteristics that are exploited herein to generate virtual pictures from the test. One can control the texture and aspects of the materials used, the intensity of lighting and the specifications of the cameras used for the rendering.

\subsection{Rendering procedure}

The visualization pipeline (Figure 5 ) is as follows. The testing machine is modeled and decomposed into different subsets in a CAD software (in this case CATIA V $5^{5}$ ) and each subset is

\footnotetext{
${ }^{5}$ https://www.3ds.com/fr/produits-et-services/catia/
} 
imported into Blender, using the .stl file format, widely used for rapid prototyping or 3D printing as it describes efficiently the surface geometry of $3 \mathrm{D}$ objects (without any representation of color or texture), thereby enabling different visual treatments for each of the subsets in Blender (in terms of material or texture properties). Different lighting sources and cameras are added to the scene. It is to be mentioned that those different steps can easily be scripted using Visual Basic (for CATIA V5) and Python (within Blender).

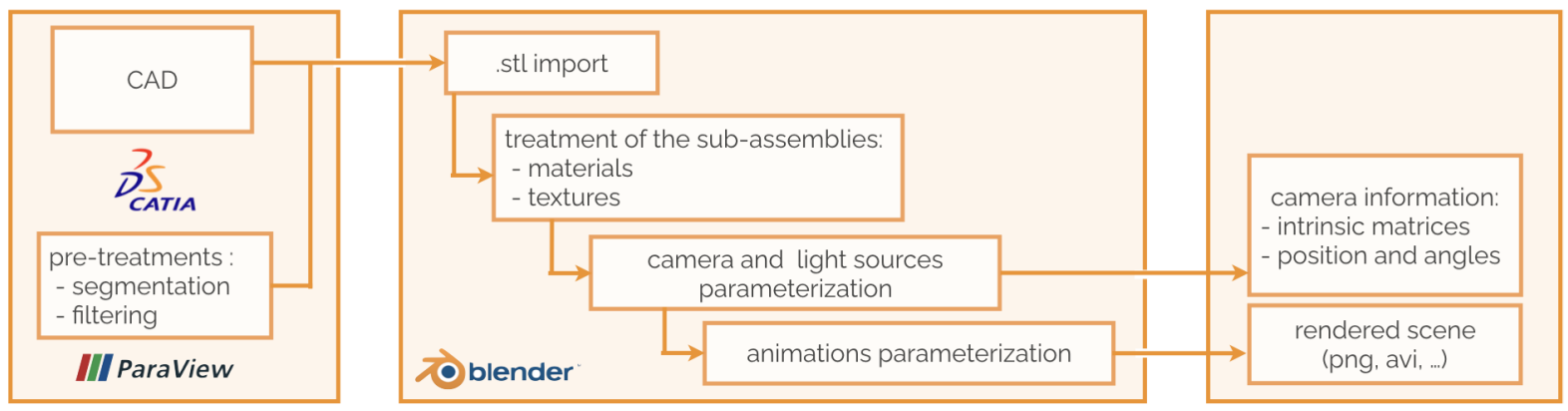

Figure 5: Visualization pipeline. From the pre-processed CAD, the geometry is imported within Blender, the virtual environment is set up, the animation is parameterized, and synthetic images are generated.

\subsection{Parameterization of the cameras}

As mentioned in the previous sections, one goal of this study is to define an adequate positioning of the different cameras. In real setups, cameras can nearly freely (yet in a time consuming manner) be moved along Newport bars surrounding the hexapod (Figure 3). Within Blender, cameras can be assigned to non-uniform rational B-splines (NURBS [40]) paths, which is described by a set of control points. The position of the cameras can be optimized along those trajectories under given constraints, such as maintaining horizontality. This point is additionally described in a further section.

\subsection{Surface patterning of beams}

To improve the rendering of the specimen, a picture of the speckle pattern was taken on the actual beam [2]. This is achieved by using the UV-mapping functionality of Blender, a technique that enables this pattern to be mapped onto the virtual sample by projecting the $2 \mathrm{D}$ image of the speckle onto 3D surfaces:

1. the speckle pattern (Figure 6(a)) is chosen or generated. This texture is treated with symmetries in order to have a smoother pattern by creating a seamless speckle;

2. the beam surface (Figure 6(b)) is "unfolded" to create a UV-map (Figure 6(c));

3. the picture of the speckle (Figure 6(b)) is overlaid over the 2D map (Figure 6(d));

4. the beam is finally "wrapped" back with the new texture (Figure 6(e)). 


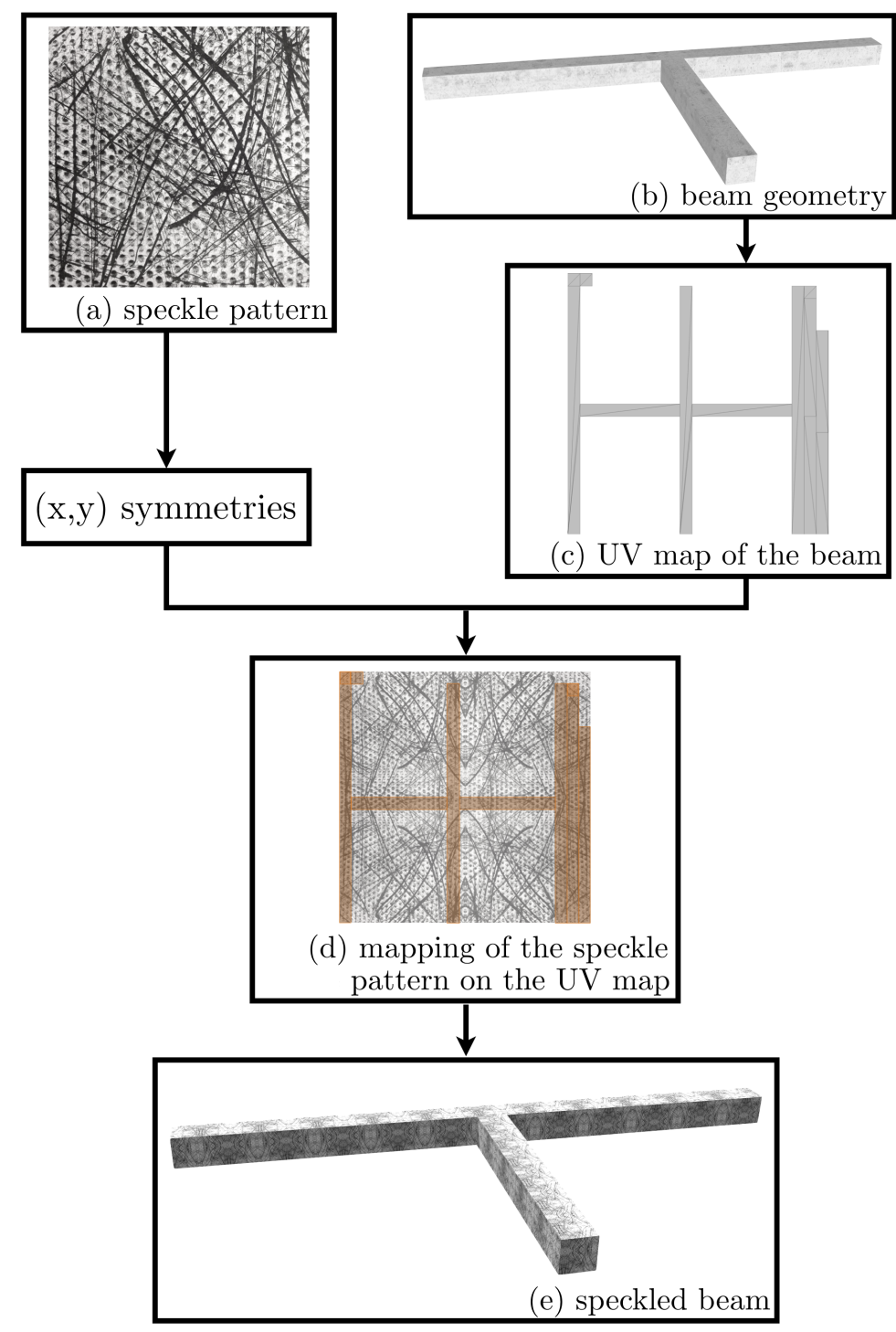

Figure 6: Stages for beam patterning in Blender. A speckle pattern is chosen or generated (a), the 3D structure (b) is unwrapped, creating a UV map (c) onto which the texture pattern (with symmetries to avoid sharp boundaries) is applied (d). The unwrapped structure is then wrapped again (e).

It is to be mentioned that by adding $x$ and $y$ symmetries to the sample on the UV map, one can create a seamless speckle, in order to avoid a large number of discontinuities on the texture when wrapping back the object.

\subsection{Surface coverage and obscuration}

This section details essential aspects of the modeling of the experiment, during which the covered subjects include the choice of the camera, its positioning in the 3D space in order to avoid possible obstructions of objects (e.g., Newport bars, lighting heads), the surface coverage of the targeted zones, the influence of lighting and the occurrence of shadows due to possible obscuration that may degrade the correlation results. In order to deal with those elements, it is assumed that the positions of the experimental device and the sample are known and that those of the cameras have to be defined along specific directions. 
Numerous parameters of the cameras can be specified in Blender such as the size of the sensor, the depth of field or the focal length of the lens. These parameters allow the surface coverage to be determined for different configurations of $\{$ camera+lens $\}$ sets. Once the initial scene is set up, it is easier for the user to make an optimal \{camera+lens\} choice and position in order to avoid obstructions from objects of the scene or hint at determining a priori the best configuration for multiview systems. Once the obstructions are avoided, one must ensure that no shadow may degrade the test.

Lighting is also an essential feature when performing digital image correlation analyses [18]. Finely modeling LED panels and setting up their position is a very important step to improve the quality of test. In the same way the cameras are positioned, light sources can be added to the virtual scene and improve the design of the experiment, keeping in mind that one must ensure that no element (light source, camera, Newport bar or even cable) will generate shadows on the areas of interest. An example (see Section 5.2.1) is shown in Figure 7, for which a Newport bar created a shadow on the area of interest (left image). A LED panel was added targeting the latter region and one can barely notice the shadow on the right image.
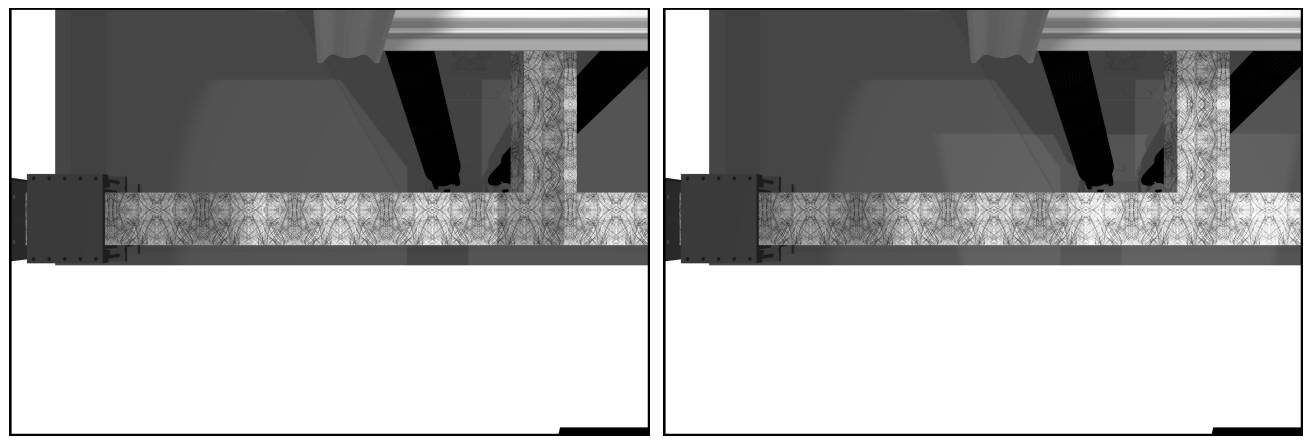

Figure 7: Shadows due to the use of Newport bars to support the cameras appear on the rendered image from camera 1 (see example Section 5.2.1) of the node area (left). The use of an additional light source (LED panel) reduces its effect (right).

Blender contains many useful features that allow light sources to be accurately described and the projected shading of the objects on the scene to be represented. For example, both direct and indirect light emissions can be taken into account, which are essential for multi-camera systems with multi-light sources and numerous possible obstructive objects. Different light sources are used, with isotropic or specific emission directions (cone, square or rectangle, very useful for modeling LED panels), or taking into account (or not) the distance falloff ${ }^{6}$. (An)isotropic shading effects can also be described with a particular emphasis being put on the interpolation between lit and shadowed areas that may induce higher uncertainties in correlation analyses.

All these features provide an accurate 3D representation of the experiment to be performed, in addition with an estimate of the level of uncertainty depending on the goal, namely, the static positioning of the cameras and light sources, the influence of the volume of given elements, the resulting obstructions and shadows, or photo-realistic rendering for goal-oriented optimization (see Section 5). Figure 8 shows the full setup of the experiment and Tables 1 and 2 give the camera specifications and the corresponding synthetic images generated with Blender following the procedure described in Figure 5.

\footnotetext{
${ }^{6}$ https://en.wikibooks.org/wiki/Blender_3D:_Noob_to_Pro
} 


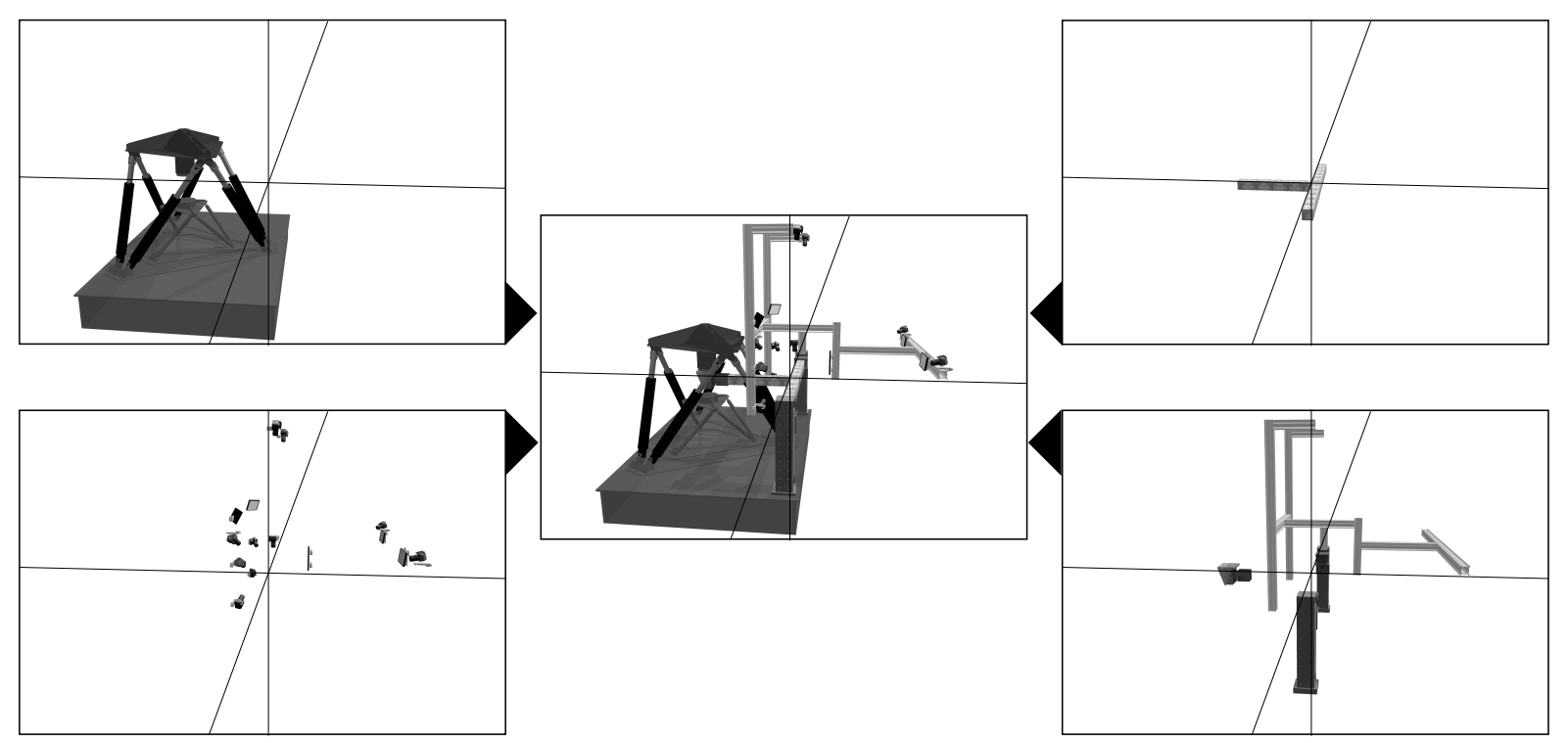

Figure 8: View of the different elements composing the rendered scene. Testing machine (top left), specimen (top right), acquisition devices and additional light sources (bottom left), and additional equipments (bottom right)

Table 1: Description of the acquisition devices. The aperture has the same value for all the cameras and is considered to be very large, the shutter speed not being an issue herein.

\begin{tabular}{c|c|c|c} 
Cam. \# & Model & Sensor model $/$ size $\left(\mathrm{mm}^{2}\right)$ & Lens $(\mathrm{mm})$ \\
\hline$(1,2)$ & Canon 45D & CMOS $22.2 \times 14.80$ & 100 \\
\hline$(3,4)$ & Canon 45D & CMOS $22.2 \times 14.80$ & 50 \\
\hline$(5,6,7)$ & Canon 60D & CMOS $22.3 \times 14.90$ & 35 \\
\hline$(8)$ & Canon 60D & CMOS $22.3 \times 14.90$ & 24 \\
\hline$(9,10)$ & Canon 45D & CMOS $22.2 \times 14.80$ & 24
\end{tabular}


Table 2: Synthetic pictures generated within Blender for the 10 cameras used in the experiment

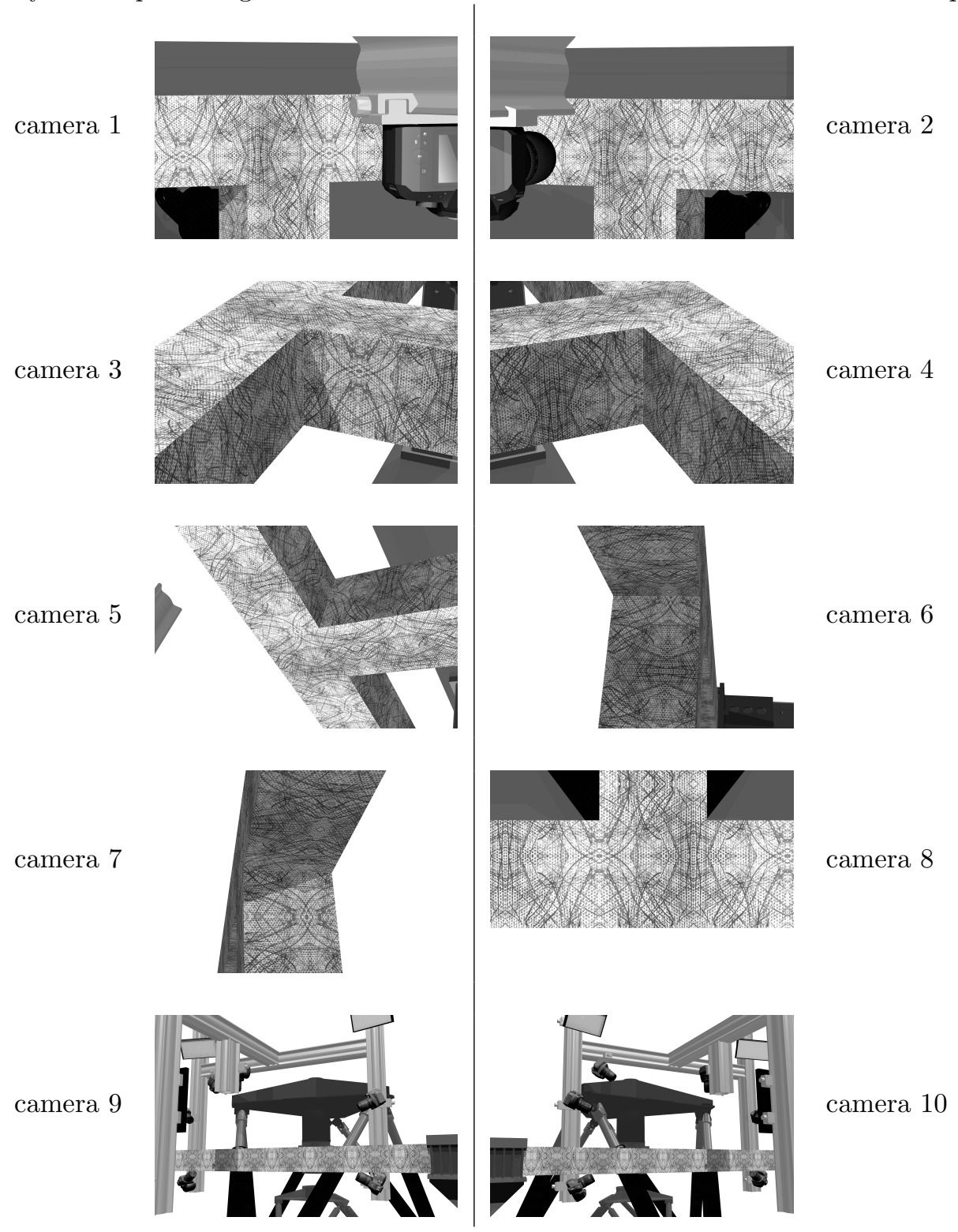

\section{Global multi-view and multi-surface correlation}

\subsection{Introduction to multi-view correlation}

Before addressing the question of the merit of "virtual design," it is necessary to recall some features of multi-view correlation, and particularly its global version used to analyze the experiment. Multi-view refers to the analysis of two or more images of the same object from different points of view in order to measure its 3D surface shape [41]. It generally relies on a first calibration step where the geometry of the acquisition system is determined (position and orientation of each camera in the $3 \mathrm{D}$ real world, parameters that are already known in the 3D virtual one), and a second step during which the shape of the specimen is analyzed. Multi-view correlation is a similar technique designed to measure the overall kinematics of the specimen $[18,41]$.

Global multi-view correlation (with a multi-camera acquisition setup) chooses as the reference a virtual 3D object with its own frame [41]. Each camera is then linked to the 3D object from 
the camera model (e.g., pinhole projection model). At least two cameras are needed, although any larger number can potentially be used, which is the case for the test presented herein. During the calibration step, the camera model parameters are determined by registering the different views onto the $3 \mathrm{D}$ virtual specimen (whose theoretical shape is known a priori, although it may slightly differ from reality). The difficulty is that the shape is known, but generally the surface pattern is not. However, the consistency of the pattern as it can be transferred from each image onto the 3D virtual object is sufficient to determine the camera geometrical parameters. Therefore, after the global correlation step, the 3D virtual model is endowed with the "real" pattern. The recourse to this 3D model dressed with the actual pattern provides a natural opportunity to assemble different camera views even with partial coverage (provided most of the surface is observed by at least two cameras, or assuming that the CAD model is reliable enough to bridge partial views). The more redundant the observation (or the larger the number of cameras and their field of view), the least uncertainty is achieved (Section 5).

For the present experiment, all geometrical quantities (shape, positions, displacements) are defined in the specimen coordinate system, which is identical for all images. Therefore, a global multi-view registration is to be carried out gathering the information of all cameras and all surfaces. A unique set of unknowns (displacement or positions for example) is then to be sought. Let us stress here the difference with local approaches where camera pairs are treated independently, choosing one camera as master, and where the final result is obtained from the optimization of the positioning of the surface shape through a dense cloud of points as a way to solve for incompatibilities [18].

\subsection{Camera calibration and shape measurement}

Camera calibration allows 3D quantities (defined in the sample coordinate system, $\mathbf{X}$ ) to be associated with 2D quantities (defined in the image space $\mathbf{x}^{i}$ ) for camera $i$ by determining the projection matrix $\left[\mathbf{M}^{i}\right][42]\left(\left[\mathbf{M}^{i}\right]\right.$ is here a matrix representation of an intrinsic object defining the projection $\mathbf{M}^{i}$ (Figure 9)

$$
\left\{\mathbf{x}^{i}\right\}=\left[\mathbf{M}^{i}\right]\{\mathbf{X}\}
$$

where $\left\{\mathbf{x}^{i}\right\}$ and $\{\mathbf{X}\}$ are the homogeneous coordinates in the camera $i$ plane, and for the considered $3 \mathrm{D}$ point, respectively. 

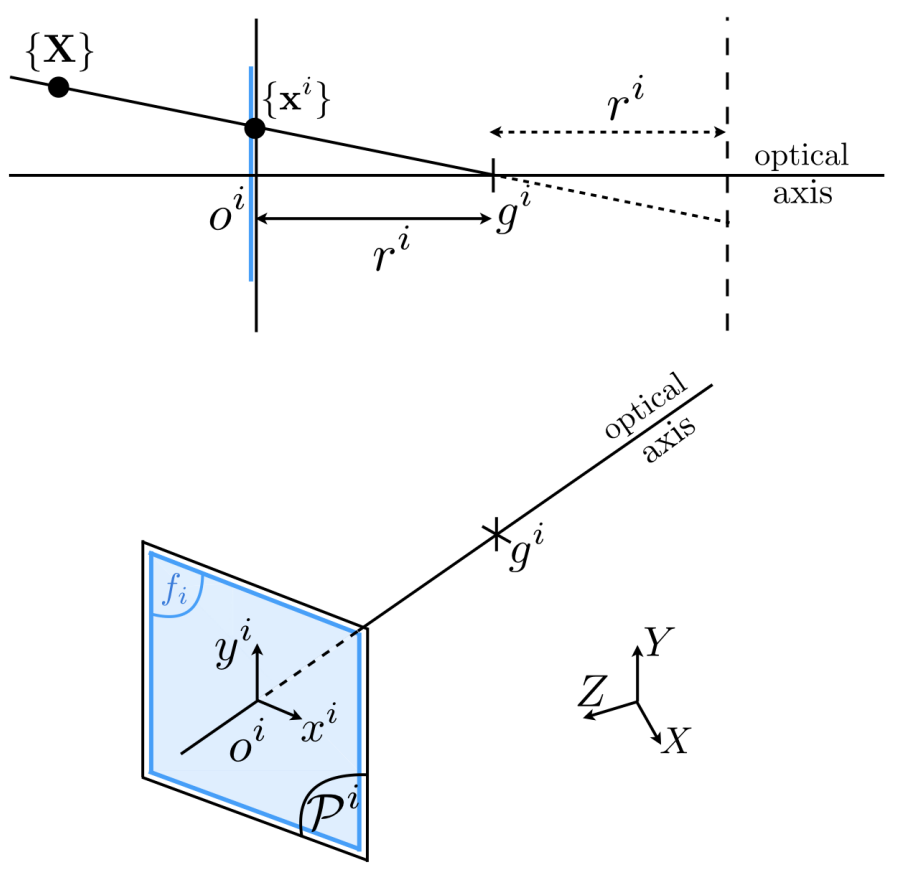

Figure 9: Front image plane model for camera $i$ and associated coordinate frames. Image symmetry with respect to $x-y$ plane through the optical center $g^{i}$ is assumed [18].

The available CAD model describes the specimen shape. In the present case, it is convenient to decompose it into a set of $n$ elementary (here planar) surfaces $\left\{n^{s}\right\}$, indexed by a surface label $s$, so that each surface has its own parametric space $\boldsymbol{\xi}^{s}$. Adjacent surfaces sharing an edge will also share control points, so that surface continuity along the edge is ensured. The CAD model relates the parametric space $\boldsymbol{\xi}^{s}$ to the $3 \mathrm{D}$ surface $\mathbf{X}\left(\boldsymbol{\xi}^{s}\right)[40]$.

As above mentioned, for global multi-view correlation [41], each camera is considered individually, calling $f^{i}$ the image captured by camera $i$, and the calibration step is performed with respect to the virtual 3D reference by considering the intrinsic pattern, which is denoted as $\hat{f}$. Because each camera may have a different sensitivity and due to its positioning observe the different faces with potentially different lighting, it is necessary to adjust individually brightness $b$ and contrast $c$ for each camera $i$ and each surface $s$. The calibration then consists in minimizing the multi-view and multi-surface functional

$$
\varrho^{2}\left(\left[\mathbf{M}^{k=1, n^{c}}\right]\right)=\sum_{s=1}^{n^{s}} \sum_{i=1}^{n^{c}}\left\|b^{i, s}+c^{i, s} f^{i}\left(\left[\mathbf{M}^{i}\right]\left\{\mathbf{X}\left(\boldsymbol{\xi}^{s}\right)\right\}\right)-\hat{f}\left(\boldsymbol{\xi}^{s}\right)\right\|^{2}
$$

with respect to all $\left[\mathbf{M}^{k}\right]$ projection matrices associated with the transformations $\mathbf{M}^{k}$. In the above expression, the sum runs over all $n^{c}$ cameras and all $n^{s}$ surfaces. Let us note that to make the problem well-posed, the mean value and the variance of $\hat{f}$ are undetermined, and one may choose conventionally to set them to 0 and 1 , respectively. The norm involves the quadratic difference in gray levels between image and virtual reference summed over the image plane of each camera $i$. If a (good enough) approximation of the projection matrices is known, which can be determined using several methods (a point detailed in Section 5), each image point $\mathbf{x}^{i}$ can be mapped onto a point $\mathbf{X}\left(\boldsymbol{\xi}^{s}\right)$ on surface $\mathrm{S}^{s}$, and hence, averaging over all images (after correcting for the appropriate brightness and contrast corrections of gray levels) observing the same physical surface provides such 
a reference object with its intrinsic pattern

$$
\hat{f}\left(\boldsymbol{\xi}^{s}\right)=\frac{1}{n^{c}} \sum_{i=1}^{n^{c}} b^{i, s}+c^{i, s} f^{i}\left(\left[\mathbf{M}^{i}\right]\left\{\mathbf{X}\left(\boldsymbol{\xi}^{s}\right)\right\}\right)
$$

which is the average over the images capturing the points $\boldsymbol{\xi}^{s}$. Furthermore, the CAD model itself can be corrected, as deviations from reality are always present. This correction can be cast into the very same framework, i.e., minimizing the multi-view functional (2), but now with respect to the control point positions of the NURBS patches (from the CAD description $[43,44]$ ).

The very same methodology can be used to measure 3D displacement fields of the specimen surfaces as they are deformed by mechanical loading [44, 41]. Displacement fields are described within the language of the CAD surface shape description and the parameterization is unique for each surface. The resulting multi-view functional to be minimized with respect to those displacements is actually very similar to Equation (2), but now the projection matrices and intrinsic pattern are set, and only the change in the surface geometry is considered. Each camera adds its contribution to the global functional with information pertaining to a partial surface coverage. The setting is not a collection of $n^{c}$ "discrete" stereo-correlation systems but rather a global multi-view and multi-surface formulation [41].

\section{A priori determination of uncertainties}

\subsection{Bridging $3 D$ rendering and multi-view correlation}

Using the previously described Blender procedure, a set of synthetic images as would be captured in the experimental setup can be generated. Those images can be made more representative of real experimental conditions by the addition of a (controllable) random noise [18, 37]. They can also be color adjusted in order to mimic different sensitivities of camera sensors with respect to color or light intensity.

These pictures can further be processed by global multi-view or stereo-correlation procedures. It is important to underline that the two codes do not share any common part, so that no bias is to be feared. A first check of the consistency of the two codes is that the known system geometry from Blender allows for a good coincidence of the images (and in particular that the intrinsic pattern of the $3 \mathrm{D}$ virtual reference computed at the first stage of the DIC procedure matches the one that was produced in Blender, although only 2D synthetic pictures were used). In particular, one could study the calibration phase where the geometry and optical parameters of the experiments are to be measured. This is usually carried out with calibration targets that are positioned (and possibly moved) in the real 3D world [18]. The processing of photo-realistic sets of images offers the opportunity to evaluate the uncertainty of this calibration phase since it can be carried out on virtual images up to the determination of the projection matrices that can be compared with their exact values.

However these two steps are time consuming as they imply a dialog between different softwares. Other much faster tools can be envisioned so as to guide the optimization of the setup based on an estimate of the displacement uncertainty. The following discussion details very low-cost operations that have been implemented within Blender as a Python script.

\subsection{Sensitivity fields}

In the above-described registration procedures, one key element that is related to the optical setup is the so-called sensitivity field $\mathbf{s}_{i}\left(\boldsymbol{\xi}^{s}\right)$ for every camera $i$, namely, the change $\delta \mathbf{x}_{i}\left(\boldsymbol{\xi}^{s}\right)$ in the 
camera plane induced by a change $\delta \mathbf{X}\left(\boldsymbol{\xi}^{s}\right)$ in the 3D position of any considered point of the surface of interest $s$

$$
\mathbf{s}_{i}\left(\boldsymbol{\xi}^{s}\right)=\frac{\partial \mathbf{x}_{i}}{\partial \mathbf{X}}\left(\boldsymbol{\xi}^{s}\right)
$$

which is expressed in the parametric space described by $\boldsymbol{\xi}^{s}$. The sensitivity fields are therefore expressed in pixel $/ \mathrm{m}$. For a given 3D point $\mathbf{X}\left(\boldsymbol{\xi}^{s}\right)$, the number of cameras that image it has to be determined. Once this information is available, the global sensitivity corresponds to the L2-norm of the vector sensitivities of each camera. From Equation (1), it is concluded that the sensitivities are related to the projection matrices $\left[\mathbf{M}^{i}\right]$. It is worth noting, however, that a linear relationship is obtained only when dealing with homogeneous coordinates. When reverting to 3D coordinates for the physical points, and 2D coordinates at the pixel level, the previous relationships are no longer linear. This is also true for their first order derivatives defining the sensitivities.

\subsubsection{Three-cameras system covering the joint}

Figure 10 shows a first test of the virtual experimental setup for which three cameras are monitoring the beam-column joint.

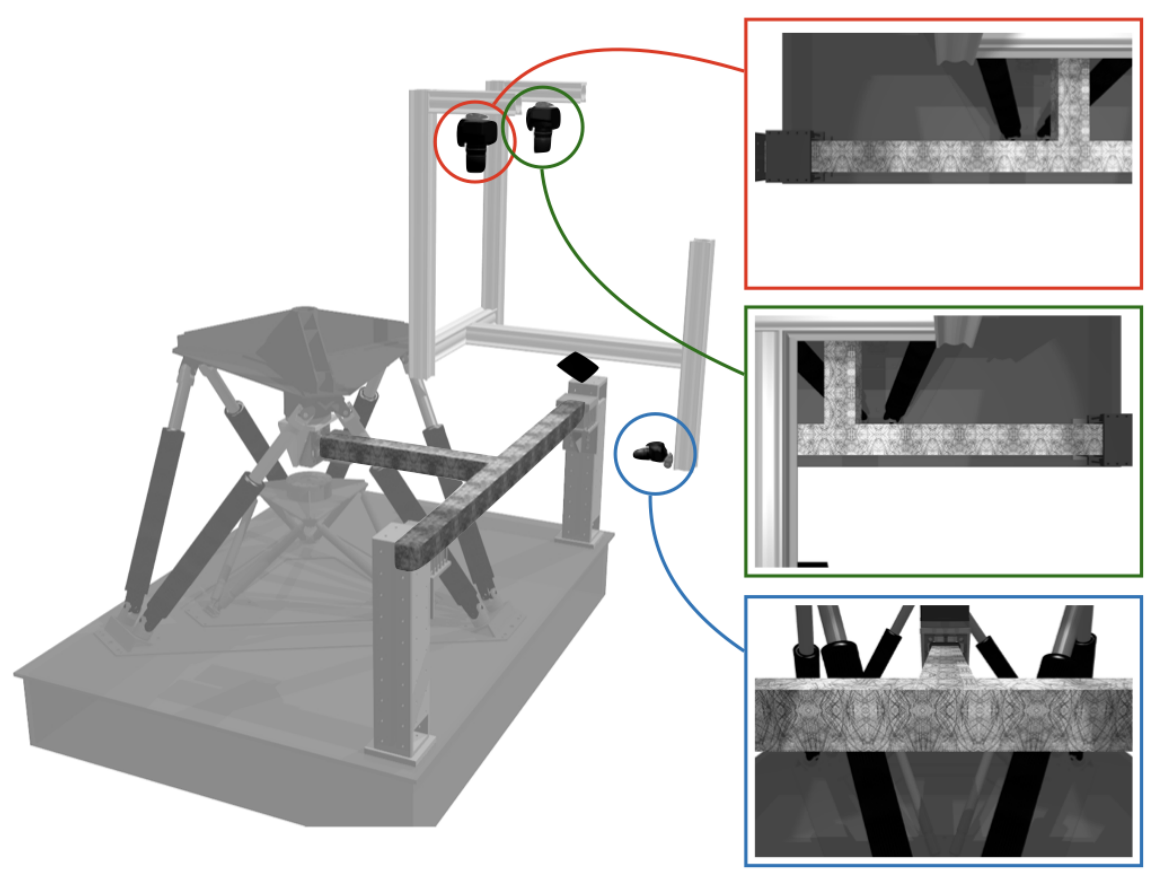

Figure 10: 3-camera system and LED lighting monitoring one part of the concrete sample (left). Synthetic images generated from the 3 cameras (right, from top to bottom: camera 1, 2 and 3).

For the remainder of the paper, the description and designation of the surfaces of the beamcolumn assembly is performed according to the first-angle multi-view projection map ${ }^{7}$ as shown in Figure 11.

\footnotetext{
${ }^{7}$ https://en.wikipedia.org/wiki/Multiview_projection\#First-angle_projection
} 


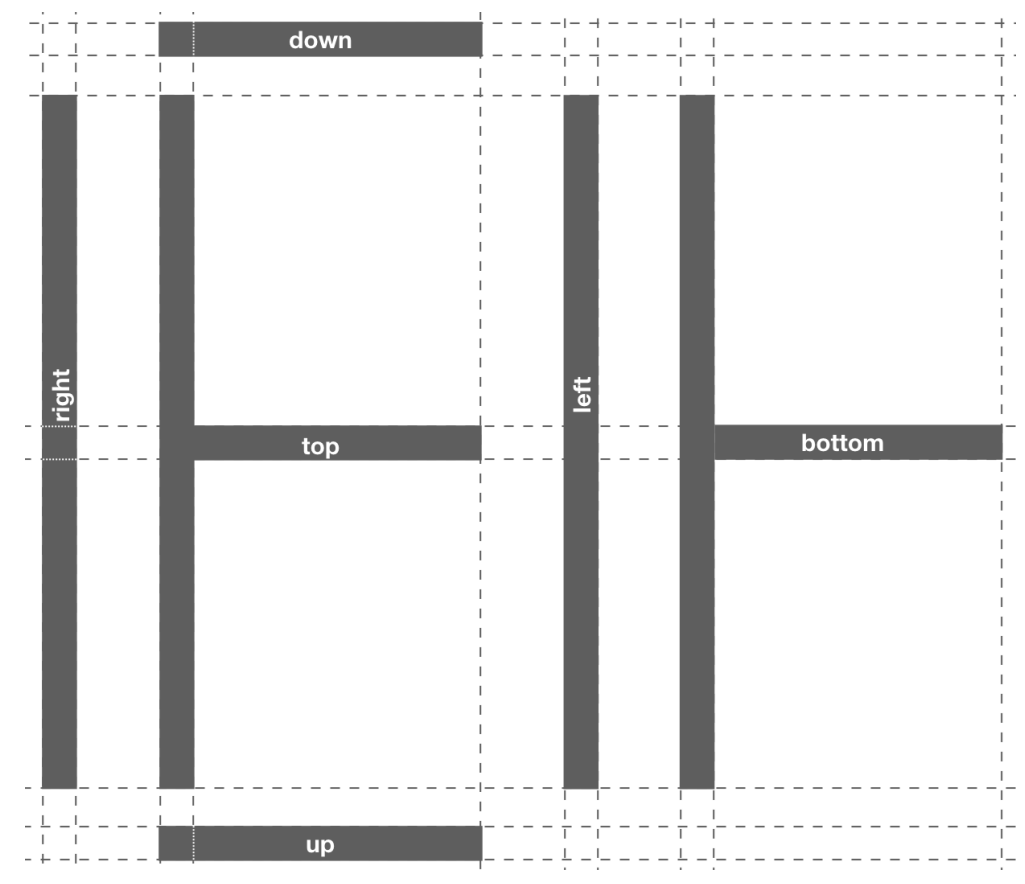

Figure 11: First-angle multi-view projection map. The points of interest are projected in the same direction as the ray of sight, onto the plane that lies behind the objects as determined by the ray of sight.

Camera 1 (respectively camera 2) is located above the sample, and covers the upper (respectively lower) part of the top surface of the beam and the beam-column joint. Camera 3 provides a much higher resolution of the latter as it located close to the joint and focuses on the connection with a $19^{\circ}$ tilt with respect to the top plane of the sample. Figure 12 shows the coverage map of the multicamera system, i.e., the number of cameras monitoring each point of the surface of the sample. It however does not take into account the intrinsic parameters of each camera, contrary to the sensitivity fields. 

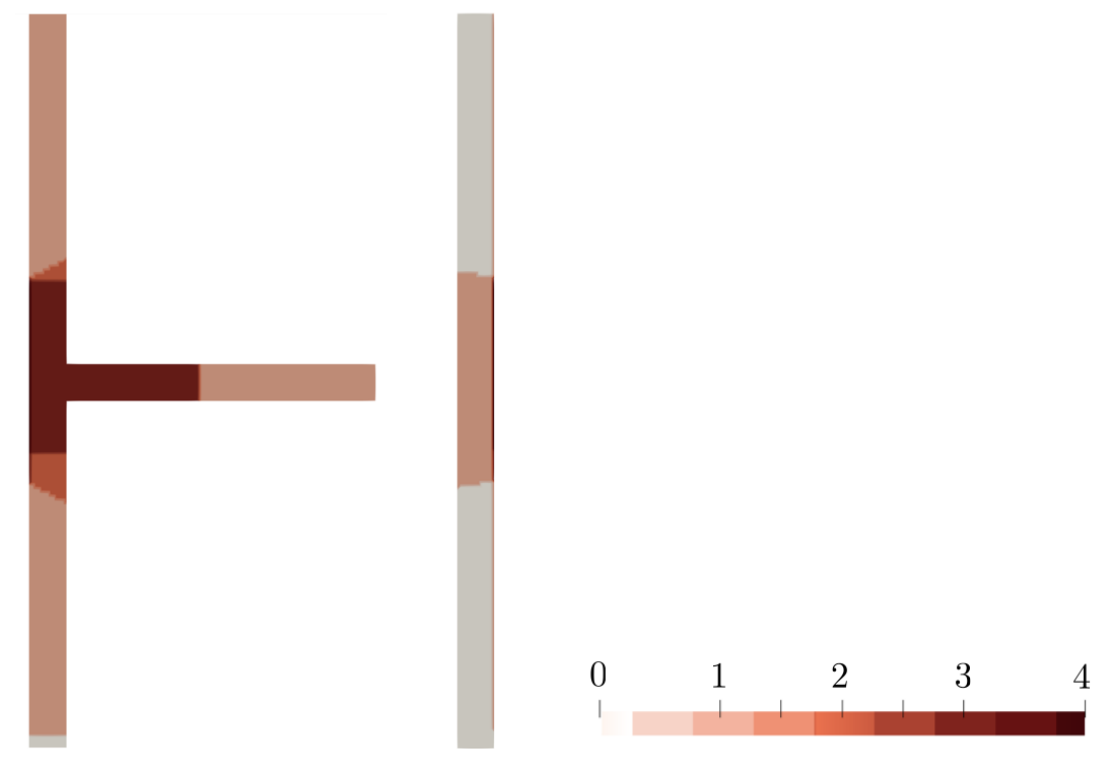

Figure 12: Coverage map of the sample (top and left surfaces, according to the projection map Figure 11). It shows the number of cameras monitoring each point of the surface.

When each camera is positioned, its projection matrix $\left[\mathbf{M}^{i}\right]$ is known. Therefore, the sensitivity fields can be computed via Equation (4) within a Python routine run in Blender. Figure 13(a) (respectively (b), (c)) shows the sensitivity field along the $X$ (respectively $Y, Z$ ) directions. More than getting these fields per camera, which are singular for a planar surface [42], it is interesting to study the sensitivity per surface observed by the different cameras. Even though cameras 1 and 2 only provide information on the top surface with a coverage of the node, camera 3 adds a more detailed input on the top surface of the node, but also on the left surface. 


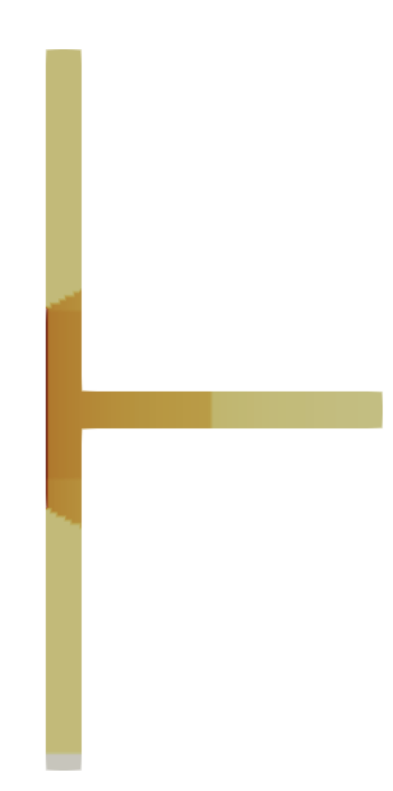

(a) Sensitivity along the $X$ direction

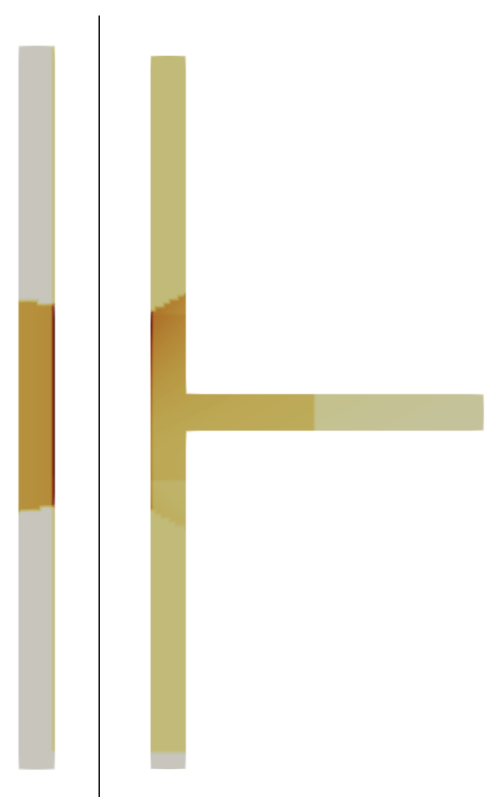

(b) Sensitivity along the $Y$ direction

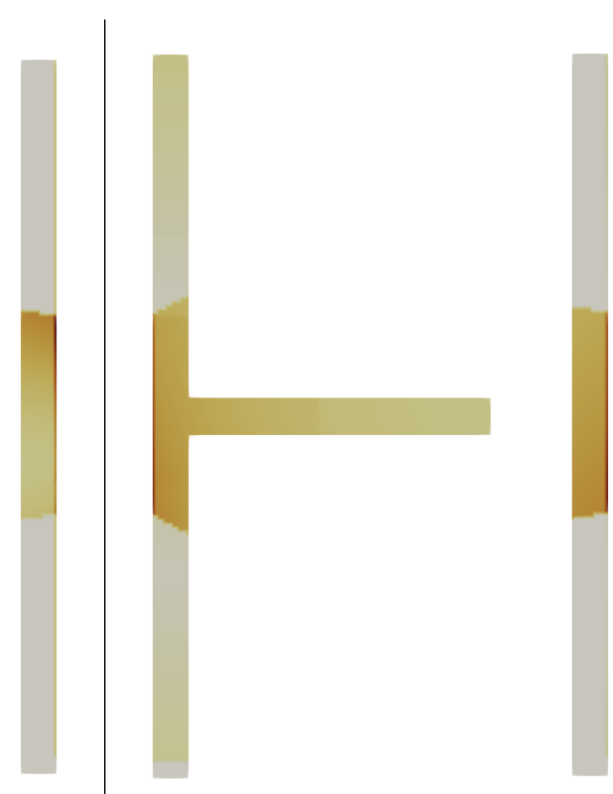

(c) Sensitivity along the $Z$ direction

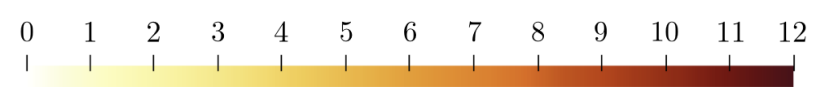

Figure 13: Sensitivity fields in the $X$ (left), $Y$ (center) and $Z$ (right) directions provided by the 3 cameras shown in Figure 10. All fields are expressed in mpixel/um. Top and left view according to the projection map defined in Figure 11.

The area of interest is here covered with multiple cameras at different resolutions, all of which increase the global sensitivity. One can notice that the best coverage of the targeted zones leads to higher sensitivity. This is an important information in the sense that the uncertainty on measured displacement fields is related to the inverse of the sensitivity fields, as will be shown in the sequel. The approach provides quantitative estimators in order to perform constrained optimization in order to position the cameras to achieve the highest sensitivities in regions of interest.

\subsubsection{Full 10-cameras system covering the entire structure}

This section shows the results for the full 10-cameras system shown on Figure 3. Figure 14(a) shows the global coverage map of the multi-camera system, with a specific close-up view on the top part of the beam-column node (Figure 14(b)) as it is the main region of interest for the study of complex damage mechanisms. This map shows that the node is covered on each of its surface by at least 3 cameras, which is more than classical stereo-systems. 


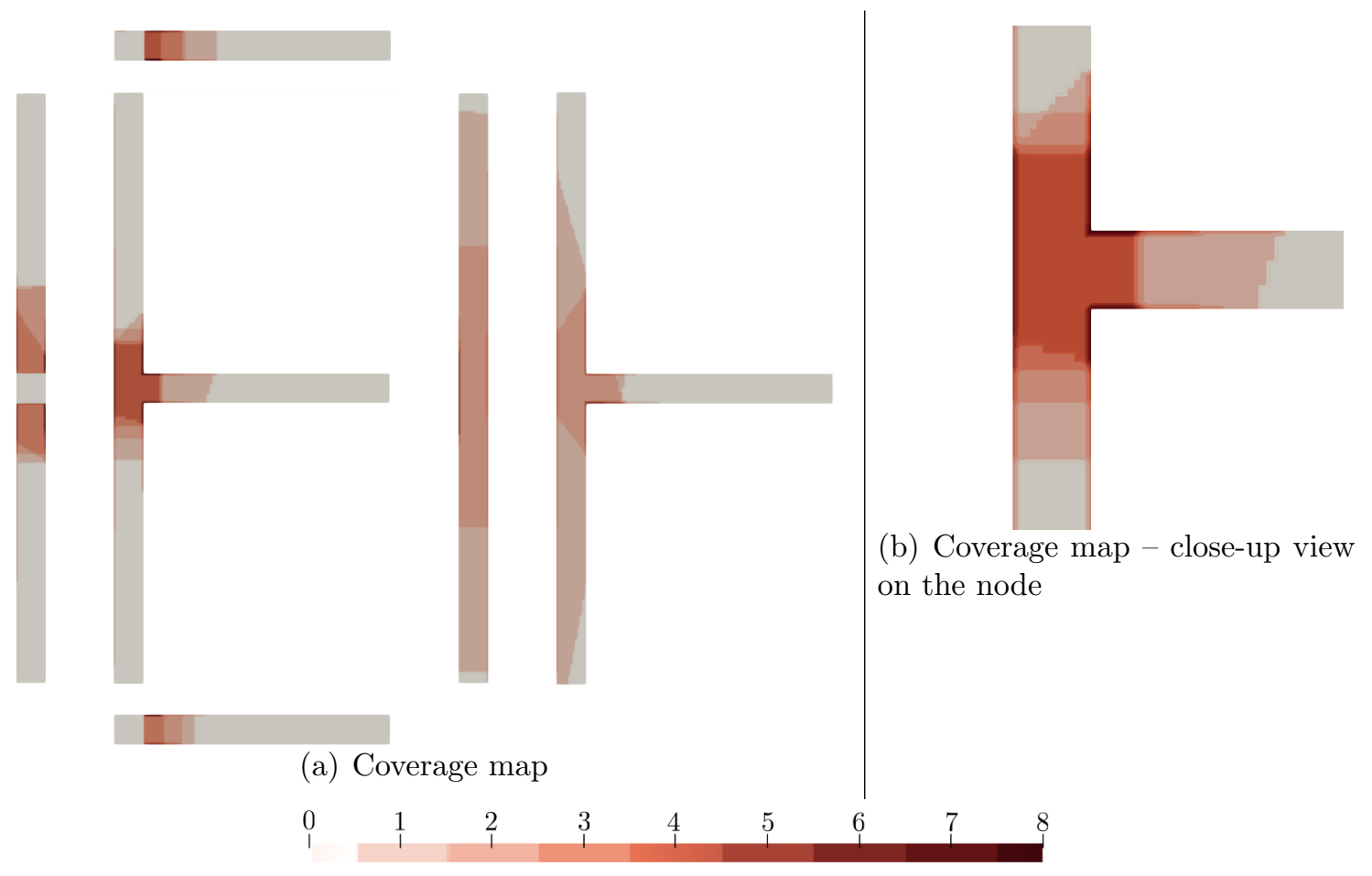

Figure 14: Coverage for the full test with 10 cameras following Figure 11 (left). Close-up view of the top surface of beam-column node (right).

Figure 15(a) (respectively (b), (c)) shows the corresponding sensitivity map along the $X$ (respectively $Y, Z$ ) directions again computed within Blender using Equation (4). The global coverage shown in Figure 14 translates well on the sensitivity map. The dense coverage of the node leads to a high sensitivity in all directions, much higher than for the first example with only 3 cameras (i.e., $30 \mathrm{mpixel} / \mathrm{\mu m}$ versus $8 \mathrm{mpixel} / \mathrm{\mu m})$. 


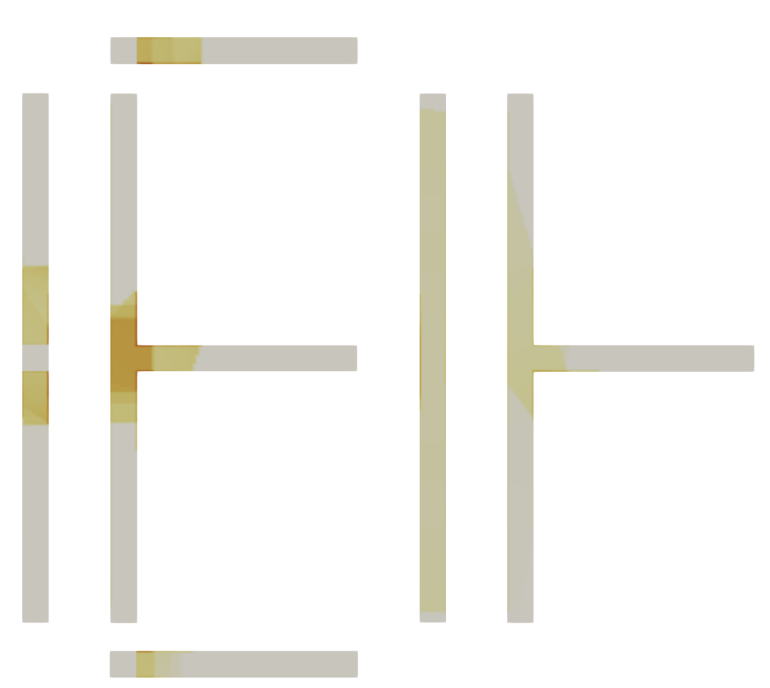

(a) Sensitivity along the $X$ direction

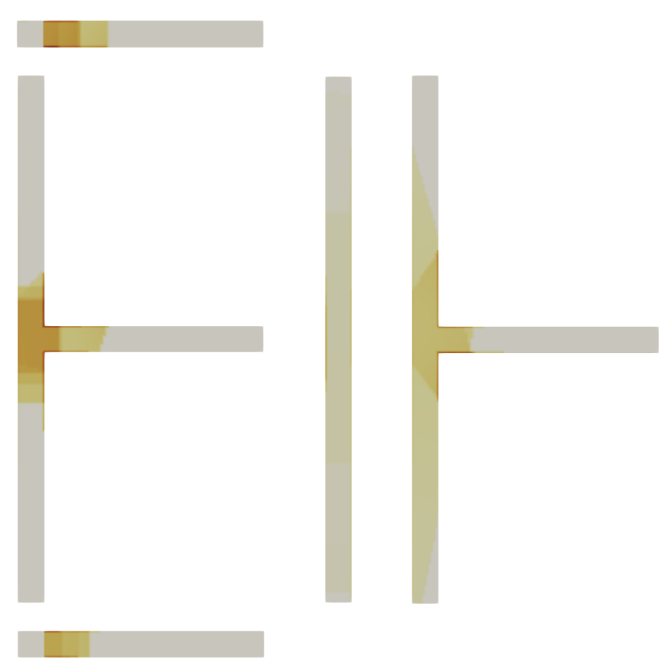

(b) Sensitivity along the $Y$ direction

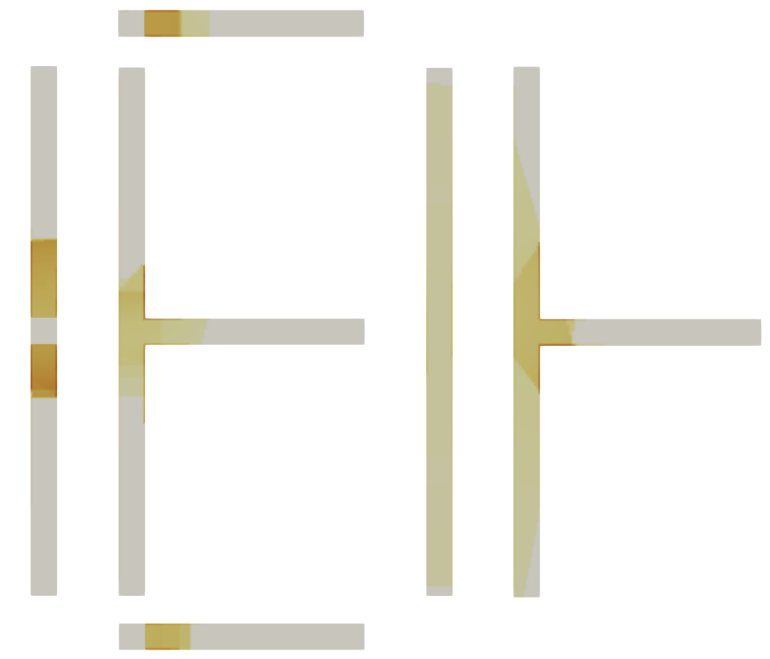

(c) Sensitivity along the $Z$ direction

$\begin{array}{lllllllll}0 & 5 & 10 & 15 & 20 & 25 & 30 & 35 & 40 \\ 1 & 1 & 1 & 1 & 1 & 1 & 1 & \perp & 1\end{array}$

Figure 15: Sensitivity fields in the $X$ (a), $Y$ (b) and $Z$ (c) directions provided by the 10 cameras shown in Figure 3. All fields are expressed in mpixel/ $\mu \mathrm{m}$. Projections follow Figure 11.

Sensitivity fields are a first step in the process of quantifying a priori the uncertainties on the displacement field. However it provides a good estimator of this quantity, it is only based on the geometry of the multi-view system with respect to the specimen as it does not take into account the pattern (speckle) and the texture of the surfaces, as well as lighting. It is to be recalled that these fields do not take into account the kinematics of the specimen during the test. In the present study, the displacement at the end of the column is about $15 \mathrm{~mm}$ in two directions as shown on Figure 4. Such displacement should not impact drastically the sensitivity maps. A way to assess that would be to develop a tool that deforms the mesh in Blenderaccording to the measured or simulated kinematics. The next section goes a step farther along the estimation of the displacement uncertainties. 


\subsection{Image pixels and gray levels}

Multi-view or stereo-correlation use the image contrast in order to measure displacement fields [18, 45]. Thus it is important to have a quantitative description of these image gradients. However, they depend on the image definition through the correspondence of pixels (from any image) to the parametric space. It is worth emphasizing that this is a key feature of photo-realistic rendering softwares.

For the sake of simplicity, let us assume that the speckle pattern in the parametric space, $\hat{f}\left(\boldsymbol{\xi}^{s}\right)$, is known with an arbitrary resolution. It was earlier noted that the image of camera $i$ would be related to the intrinsic pattern $\hat{f}$ thanks to a brightness $b^{i, s}$ and contrast $c^{i, s}$ adjustment that depends on camera $i$ and the observed surface $s$ (for punctual light sources and/or curved surfaces these brightness and contrast terms may have a spatial modulation, but here only flat surfaces are considered, potential long wavelength variations will be ignored, and thus $b^{i, s}$ and $c^{i, s}$ are assumed uniform), hence $f^{i}\left(\mathbf{x}^{i}\right)=b^{i, s}+c^{i, s} \hat{f}\left(\boldsymbol{\xi}^{s}\left(\mathbf{x}^{i}\right)\right)$.

However, for DIC purposes, the speckle pattern is designed to have sharp gradients at very small scales. The physical effect of pixel discretization is mimicked by a regular sampling not of $\tilde{f}^{i}\left(\mathbf{x}^{i}\right)=b^{i, s}+c^{i, s} \hat{f}\left(\boldsymbol{\xi}^{s}\left(\mathbf{x}^{i}\right)\right)$ but rather of a convolution of this speckle pattern transported on the image plane $\widetilde{f}^{i}\left(\mathbf{x}^{i}\right)$ with the shape function of the pixel. When a high degree of regularity (e.g., cubic spline) is used for sub-pixel displacement, it is natural to use a smooth shape function, and a Gaussian filter reveals to be very convenient and accurate. This Gaussian filter contains the physical scale of the pixel. This convolution can also be transported in the parametric space through the $\boldsymbol{\xi}(\mathbf{x})$ mapping. It is useful to introduce the Jacobian of the mapping

$$
\mathbf{A}=\frac{\partial \mathbf{x}}{\partial \boldsymbol{\xi}}
$$

so that, locally, the Gaussian convolution filter reads

$$
G_{p}(\boldsymbol{\xi})=\frac{\operatorname{det}(\mathbf{A})}{2 \pi} \exp \left(-(1 / 2) \boldsymbol{\xi}^{\top} \mathbf{A}^{\top} \mathbf{A} \boldsymbol{\xi}\right)
$$

The symmetric tensor $\mathbf{A}^{\top} \mathbf{A}$ can be diagonalized. It defines two eigen-directions $\mathbf{e}_{\mathbf{1}}$ and $\mathbf{e}_{\mathbf{2}}$ with eigenvalues $a_{1}^{2}$ and $a_{2}^{2}$, respectively. When transported in the parametric space, the pixel becomes an anisometric surface element that can be approached by its geometric moment of inertia, with semi-axis $a_{i}$, in the eigen-direction $\mathbf{e}_{\mathbf{i}}$. When the surface is observed with a grazing incidence by camera $i$, one such axis, say $a_{1}$, becomes large. Similarly, along this direction, the speckle pattern is coarsened to scale $a_{1}$ and hence if a high-frequency speckle was initially dealt with, details are averaged out.

Equipped with the above tools, the way displacements are measured can now be addressed. The sensitivity $\mathbf{s}^{i}$, as defined in Equation (4), translates into a 3D displacement vector $\mathbf{d} \mathbf{U}$ and into its $2 \mathrm{D}$ manifestation in camera $i, \mathbf{d} \mathbf{u}^{i}$. The previous transfer of speckle pattern allows the gray level gradients to be computed

$$
\nabla_{\mathbf{x}} f^{i}=c^{i, s} \mathbf{A}^{-1} \nabla_{\boldsymbol{\xi}}\left[G_{p}(\boldsymbol{\xi}) \star \hat{f}(\boldsymbol{\xi})\right]
$$

where $\star$ stands for the convolution product. Thus, the change in gray level at position $\mathbf{x}$ (surface $s$ ) in camera plane $i$ due to the displacement $\mathbf{d} \mathbf{U}$ writes

$$
\mathbf{B}^{i, s} \cdot \mathbf{d} \mathbf{U} \equiv \nabla_{\mathbf{x}} f^{i} \cdot \mathbf{s}^{i} \cdot \mathbf{d} \mathbf{U}
$$

From the latter expression, the uncertainty due to a white Gaussian noise of variance $\left(\sigma^{i}\right)^{2}$ is characterized by the inverse of its covariance matrix that reads

$$
\mathbf{C}^{i, s} \equiv\left(\sigma^{i}\right)^{-2} \mathbf{B}^{i, s \top} \mathbf{B}^{i, s}
$$


Furthermore, when different cameras are assembled together for multi-view correlation, these inverse covariance matrices $\mathbf{C}^{i, s}$ are added when noise is uncorrelated between cameras. The computation of $\mathbf{C}^{i, s}$ is thus a very powerful tool as it allows for an easy assembly of different cameras within a framework where they speak the same language, and where an appropriate weight is given to each camera, accounting for its noise level, distance, orientation with respect to the surface of interest. Let us emphasize that as the surface gets to the limit of grazing incidences, the gradient of texture along this grazing direction projected onto the surface vanishes, and so does its weight, so that the transition to non-visible faces is gradual and comes in naturally.

\subsection{Mean-field estimate of the uncertainty}

It is possible to proceed one step farther and propose a mean field estimate of the previous inverse covariance so that the speckle pattern dependence can be precomputed and replaced by its statistical average (denoted with angular brackets, $\langle\square\rangle$ ). The expectation value of the gray level gradients needs to be evaluated for different short scale cut-offs. The speckle pattern is assumed to be isotropic for the sake of simplicity. However, a realistic pattern may be taken into account as it is important to have a multiplicity of scales being present so that some signal remains even when the image is blurred because of a coarse effective pixel size in the parametric space. Therefore, the expectation value of the squared modulus of the texture gradient is computed after convolution by an isotropic Gaussian filter of width $\ell$ as a function of $\ell, g_{f}(\ell) \equiv(1 / 2)\left\langle\left(\nabla G_{\ell} \star \hat{f}\right)^{2}\right\rangle$.

This quantity may now be used to compute the expectation value of the dyadic (tensor) product

$$
\begin{aligned}
\mathbf{T}^{i, s} & \equiv\left\langle\nabla_{\mathbf{x}} f^{i} \otimes \nabla_{\mathbf{x}} f^{i}\right\rangle \\
& =\left(c^{i, s}\right)^{2}\left(\frac{g_{f}\left(a_{1}\right)}{a_{1}^{2}} \mathbf{e}_{1} \otimes \mathbf{e}_{1}+\frac{g_{f}\left(a_{2}\right)}{a_{2}^{2}} \mathbf{e}_{2} \otimes \mathbf{e}_{2}\right)
\end{aligned}
$$

and from the latter, the inverse covariance assumes the following simple expression

$$
\left\langle\mathbf{C}^{i, s}\right\rangle=\left(\frac{c^{i, s}}{\sigma^{i}}\right)^{2} \mathbf{s}^{i} \cdot \mathbf{T}^{i} \cdot \mathbf{s}^{i}
$$

With this expression, any flat surface of the studied domain is characterized with the $3 \times 3$ matrix $\mathbf{C}^{i, s}$ coming from all cameras $i$ that can see the zone. With one camera, the rank of this matrix is only 2 , and hence the uncertainty is infinite along one direction (i.e., that of the optical axis). However, when several cameras are used to monitor the same surface, generically the sum of all $\mathbf{C}^{i, s}$ matrices becomes symmetric, positive and definite. Therefore the uncertainty that results from a particular camera setup (due to image noise) can be quickly computed and hence the optimization of the setup is easily accessible once add-on tools are implemented as they were for the sensitivity fields.

Let us note that only the uncertainty coming from image noise is considered in this section. Other sources of uncertainty are present (e.g., pixel interpolation, projection matrix errors, distortions) but are not discussed herein.

\section{Discussion}

Before concluding on the various aspects highlighted in this paper, it appears to be important to open a discussion about the results presented herein. What was aimed at lied at a rather methodological level, a new paradigm in the field of experimental mechanics, as it is believed that the use of photo-realistic imaging softwares, main ingredient of what was introduced as virtual experimental design, will become an essential step (not to say mandatory) in the near future as a preparation tool 
for complex experiments monitored with many cameras. This thought comes from the observation that the geometries of the objects to be studied become ever larger, and very often are $3 \mathrm{D}$ and thus require the use of a growing number of cameras [46]. The complexity of the experiment is then also increasing and the use of virtual testing is a powerful tool to reduce the time of preparation of such experiments.

The software Blender used in this work shows great advantages for this step. Its first main ingredient is that it is free and open-source, which is believed to be extremely important for academic research (the authors acknowledge that Maya or CATIAV5 could perform the same renderings, with a much higher cost for the first one and rendering time - and options - for the second). It is also to be mentioned that its development relies on a huge community of users and developers, which is a great advantage for developing new tools such as those mentioned herein. The most useful feature of Blender is probably its Python interface, which allows for process scripting (as a way to automate the rendering procedure), extraction and computation of the different parameters or operators (such as the pixel size or the projection matrices) in order to perform goal-oriented optimization. All these features provide a priori elements for a better preparation of the experiment, as well as time savings in the set up of such complex mechanical tests.

\section{Conclusion and outlook}

One of the most remarkable features of full-field measurements is their ability to quantify deformations such as those resulting either from a complex shape and/or loading history or from a constitutive law leading to localized phenomena (e.g., instabilities or fracture). Experiments can be classified into two categories, namely, those aiming at the calibration of constitutive laws of a specific material and those reproducing in-service conditions for a complete industrial part. The first category seeks the most uniform stress/strain state so that either local (e.g., strain gauge) or global (e.g., extensometer) measurement devices were sufficient to characterize strains and stresses (thanks to load cells) in the region of interest. The advent of full-field measurement techniques allowed for complex sample shape to be characterized in a complete fashion, so that instead of a simple uniformity assumption, analyses can be used to "read" displacement fields and evaluate unknown components of the constitutive law, and additionally validate the numerical model and assess its relevance for the analyzed test. This opens up the possibility of using in-service tests in order to quantify and validate the selected model for the part of interest. This is a major change of perspective for experimental mechanics, which requires the development of new tools.

As soon as the specimen shape is complex, or when symmetry is broken down by an instability (be it due to geometry or to the deformation mechanism itself), displacements have to be characterized in $3 \mathrm{D}$. An exhaustive characterization requires that any surface element where displacements are to be measured should be observed by at least two cameras (or two viewpoints if the imaging system is moving [47]). Moreover, as grazing incidence gives a very poor determination, one may restrict the considered cameras observing a surface element only by those whose angle with the surface normal is less than a prescribed level. This criterion is a natural extension of the obscuration criterion. Therefore, in order to observe a given shape over its entire surfaces, a minimum of four cameras are needed, even for a simple (convex) surface. Even if it can be rightly argued that a model may allow for an appropriate interpolation for those regions where not enough viewpoints are accessible, having a global and redundant coverage is recommended. Hence, the positioning of the cameras and the calibration of the geometrical model that is needed in order to use all cameras simultaneously becomes key to the success of the mechanical test. It was shown herein that photo-realistic tools could be instrumental to achieve such goals.

The above discussion, which was applied to the considered example, has a much broader scope 
of relevance. Because this is a clear trend in experimental mechanics, it is of high importance to recognize it as such, and develop suited strategies to not only avoid unadapted test conditions, but rather to optimize the setup to benefit from the potentialities of full-field measurement capabilities in particular in a global framework of analysis.

Most of the proposed tools were unfortunately ready only after completion of the test, and although the methodology is now available, it was not used further than to assess the uncertainty of the kinematic measurements, and hence not for optimization.

It is the authors' conviction that accounting for uncertainties and minimizing them is the way to optimize mechanical tests in the future where accessing rich and redundant information will be the rule rather than the exception. Thus, it is essential to be able to predict in a trustful way the uncertainty that will result from the setup and the analyzing tools. Along this route, 3D rendering softwares, at the heart of the methodology proposed herein, are extremely valuable instruments to pre-design and optimize experimental setups before they effectively exist.

\section{References}

[1] J.-E. Dufour, S. Leclercq, J. Schneider, S. Roux, and F. Hild, "3D surface measurements with isogeometric stereocorrelation: Application to complex shapes," Optics and Lasers in Engineering, vol. 87, pp. $146-155,2016$.

[2] A. E. Iskef, Technologies informatiques pour l'étude du comportement expérimental et numérique d'un assemblage poutre-poteau en béton armé. PhD thesis, ENS Cachan, 2016.

[3] S. Pampanin, G. Calvi, and M. Moratti, "Seismic Behaviour of RC Beam-Column Joints Design for Gravity Loads," in 12th European Conference on Earthquake Engineering, no. 726, 2002.

[4] S. J. Hamil, Reinforced Concrete Beam-Column Connection Behaviour. Phd thesis, 2000.

[5] S.-J. Hwang and H.-J. Lee, "Analytical Model for Predicting Shear Strengths of Exterior Reinforced Concrete Beam-Column Joints for Seismic Resistance," ACI Structural Journal, vol. 96, no. 5, pp. 846-858, 1999.

[6] G. Kotsovou and H. Mouzakis, "Seismic behaviour of RC external joints," Magazine of Concrete Research, vol. 63, no. 4, pp. 247-264, 2011.

[7] J. Kim and J. LaFave, "Joint shear behavior of reinforced concrete beam-column connections subjected to seismic lateral loading," NSEL Report Series, NSEL-020, 2009.

[8] B. Richard, S. Cherubini, F. Voldoire, P.-E. Charbonnel, T. Chaudat, S. Abouri, and N. Bonfils, "Smart 2013: Experimental and numerical assessment of the dynamic behavior by shaking table tests of an asymmetrical reinforced concrete structure subjected to high intensity ground motions," Earthquake Engng. Struct. Dyn.,, vol. 109, pp. 99-116, 2016.

[9] K. J. Elwood and J. P. Moehle, "Shake Table Tests and Analytical Studies on the Gravity Load Collapse of Reinforced Concrete Frames," tech. rep., Pacific Earthquake Engineering Research Center, University of California, Berkeley, California, 2003.

[10] L. N. Lowes and A. Altoontash, "Modeling reinforced-concrete beam-column joints subjected to cyclic loading," Journal of Structural Engineering, vol. 129, no. 12, pp. 1686-1697, 2003. 
[11] J. Mazars, Y. Berthaud, and S. Ramtani, "The unilateral behaviour of damaged concrete," Engineering Fracture Mechanics, vol. 35, no. 415, pp. 629-635, 1990.

[12] M. Vassaux, B. Richard, F. Ragueneau, and A. Millard, "Regularised crack behaviour effects on continuum modelling of quasi-brittle materials under cyclic loading," Engineering Fracture Mechanics, vol. 149, pp. 18 - 36, 2015.

[13] T. T. H. Nguyen, F. Ragueneau, D. Bahon, and N. Ruaux, "Macroscopic modeling of reinforced concrete joints: Applications to thermal break elements subject to earthquake loadings," Engineering Structures, vol. 79, pp. 131-141, 2014.

[14] S. A. Mahin and P. B. Shing, "Pseudodynamic Method for Seismic Testing," Journal of Structural Engineering, vol. 111, pp. 1482-1503, 1985.

[15] P. Pegon and A. V. Pinto, "Pseudo-dynamic testing with substructuring at the ELSA laboratory," Earthquake Engng. Struct. Dyn.,, vol. 29, no. 4, pp. 905-925, 2000.

[16] P. Pegon and G. Magonette, "Continuous PsD testing with non-linear substructuring: presentation of a stable parallel inter-field procedure," in JRC special publication, no. I.02.167, 2002.

[17] F. Hild, S. Roux, N. Guerrero, M. E. Marante, and J. Florez-Lopez, "Calibration of constitutive models of steel beams subject to local buckling by using Digital Image Correlation," Eur. J. Mech. A/Solids, vol. 30, pp. 1-10, 2011.

[18] M. A. Sutton, J. J. Orteu, and H. Schreier, Image correlation for shape, motion and deformation measurements: Basic Concepts, Theory and Applications. New York, NY (USA): Springer, 2009.

[19] J. C. Passieux, F. Bugarin, C. David, J. N. Périé, and L. Robert, "Multiscale displacement field measurement using digital image correlation: Application to the identification of elastic properties," Experimental Mechanics, vol. 55, no. 1, pp. 121-137, 2014.

[20] F. Hild, S. Roux, R. Gras, N. Guerrero, M. E. Marante, and J. Flórez-López, "Displacement Measurement Technique for Beam Kinematics," Optics and Lasers in Engineering, vol. 47, pp. 495-503, 2009.

[21] A. Carpiuc-prisacari, M. Poncelet, K. Kazymyrenko, H. Leclerc, and F. Hild, "A complex mixedmode crack propagation test performed with a 6-axis testing machine and full-field measurements," Engineering Fracture Mechanics, vol. 176, pp. 1-22, 2017.

[22] H. How, "Illustrated interviews, $N^{\circ} 4$. Mr. W. S. Gilbert," The Strand Magazine, vol. 2, pp. 330$341,1891$.

[23] E. DiGiulio, "Two special lenses for Barry Lyndon," American cinematographer, 2018.

[24] Blender Online Community, Blender - a 3D modelling and rendering package. Blender Foundation, 2018.

[25] Nuclear Energy Agency, "Experimental facilities for earthquake engineering simulation worldwide. are large testing facilities for nuclear power plants design and verification at risk?," No. NEA/CSNI/R(2004)10, 2004. 
[26] V. Saouma, G. Haussmann, D.-H. Kang, and W. Ghannoum, "Real-time hybrid simulation of a nonductile reinforced concrete frame," Journal of Structural Engineering, vol. 140, no. 2, p. 04013059, 2014.

[27] A. Stokes, M. Gardner-Morse, D. Churchill, and J. P. Laible, "Measurement of a spinal motion segment stiffness matrix," J. Biomech., vol. 35, pp. 517-521, 2002.

[28] F. Motoyoshi, M. Takanori, I. Tadashi, K. Takaya, K. Yuichi, and I. Satoru, "Development of 6-Axis Material Tester for Measuring Mechanical Spine Properties," J. Robot. Mechatron., vol. 18, no. 2, pp. 160-166, 2006.

[29] J. G. Michopoulos, J. C. Hermanson, and A. Iliopoulos, "Towards a recursive hexapod for the multidimensional mechanical testing of conmposites," in International Design Engineering Technical Conferences $\&$ Computers and Information in Engineering Conference IDETC/CIE 2010, 2010.

[30] B. Ding, R. M. Stanley, B. S. Cazzolato, and J. Costi, "Real-time FPGA Control of a Hexapod Robot for 6-DOF Biomechanical Testing," in 37th Annual Conference on IEEE Industrial Electronics Society, 2011.

[31] M. Ismail, "Novel hexapod-based unidirectional testing and FEM analysis of the RNC isolator," Struct. Cont. Health Monit., vol. 23, no. 6, pp. 894-922, 2016.

[32] A. Carpiuc-Prisacari, M. Poncelet, K. Kazymyrenko, F. Hild, and H. Leclerc, "Comparison between experimental and numerical results of mixed-mode crack propagation in concrete: Influence of boundary conditions choice," Cem. Concr. Res., vol. 100, pp. 329-340, 2017.

[33] A. Colson, Conditions aux limites de liaisons et d'assemblages en mécanique des structures métalliques. Thèse d'Etat (in French), Université Pierre et Marie Curie, 1984.

[34] J. Lemaitre and R. Desmorat, Engineering damage mechanics: ductile, creep, fatigue and brittle failures. Berlin (Germany): Springer Science \& Business Media, 2005.

[35] J. Flórez-López, M. E. Marante, and R. Picón, "Fracture and Damage Mechanics for Structural Engineering of Frames: State-of-the-Art Industrial Applications," IGI Global, 2014.

[36] P. Mata, A. H. Barbat, and S. Oller, "Two-scale approach for the nonlinear dynamic analysis of RC structures with local non-prismatic parts," Eng. Struct., vol. 30, no. 12, pp. 3667-3680, 2008.

[37] F. Hild and S. Roux, "Digital Image Correlation," in Optical Methods for Solid Mechanics. A Full-Field Approach (P. Rastogi and E. Hack, eds.), (Weinheim (Germany)), pp. 183-228, Wiley-VCH, 2012.

[38] B. Dasgupta and T. Mruthyunjaya, "The stewart platform manipulator: a review," Mechanism and Machine Theory, vol. 35, no. 1, pp. 15-40, 2000.

[39] F. J. Vecchio and M. P. Collins, "The modified compression-field theory for reinforced concrete elements subjected to shear.," ACI Journal, vol. 83, no. 2, pp. 219-231, 1986.

[40] L. Piegl and W. Tiller, The NURBS Book - 2nd Edition. Springer, 1997. 
[41] J.-E. Dufour, F. Hild, and S. Roux, "Shape, Displacement and Mechanical Properties from Isogeometric Multiview Stereocorrelation," J. Strain Analysis, vol. 50, no. 7, pp. 470-487, 2015.

[42] O. Faugeras, Three-dimensional computer vision: a geometric viewpoint. Cambridge, MA (USA): MIT Press, 1993.

[43] B. Beaubier, J. E. Dufour, F. Hild, S. Roux, S. Lavernhe-Taillard, and K. Lavernhe-Taillard, "CAD-based calibration of a 3D-DIC system: Principle and application on test and industrial parts," Experimental Mechanics, vol. 54, no. 3, pp. 329-341, 2014.

[44] J.-E. Dufour, B. Beaubier, F. Hild, and S. Roux, "CAD-based displacement measurements. Principle and first validations," Experimental Mechanics, vol. 55, no. 9, pp. 1657-1668, 2015.

[45] F. Hild and S. Roux, "Comparison of local and global approaches to digital image correlation," Experimental Mechanics, vol. 52, no. 9, pp. 1503-1519, 2012.

[46] K. Cosseron, R. Gras, D. Mellé, J. F. Diebold, F. Hild, and S. Roux, "Inverse Identification of the Loading Applied by a Tire on a Landing Gear Wheel," in Residual Stress, Thermomechanics 8 Infrared Imaging, Hybrid Techniques and Inverse Problems (A. Baldi, S. Quinn, X. Balandraud, J. Dulieu-Barton, and S. Bossuyt, eds.), vol. 7, pp. 13-16, Springer, Cham, 2019.

[47] I. Jovančević, S. Larnier, J. J. Orteu, and T. Sentenac, "Automated exterior inspection of an aircraft with a pan-tilt-zoom camera mounted on a mobile robot," J. Electron. Im., vol. 24, no. 6, p. 61110, 2015. 